\title{
Efficient Inclusion for a Class of XML Types with Interleaving and Counting
}

\author{
Dario Colazzo \\ Université Paris Sud, UMR CNRS 8623, Orsay F-91405 - France \\ Giorgio Ghelli \\ Dipartimento di Informatica - Università di Pisa - Italy \\ Carlo Sartiani \\ Dipartimento di Informatica - Università di Pisa - Italy
}

\begin{abstract}
Inclusion between XML types is important but expensive, and is much more expensive when unordered types are considered. We prove here that inclusion for XML types with interleaving and counting can be decided in polynomial time in presence of two important restrictions: no element appears twice in the same content model, and Kleene star is only applied to disjunctions of single elements.

Our approach is based on the transformation of each such content model into a set of constraints that completely characterizes the generated language. We then reduce inclusion checking to constraint implication. We exhibit a quadratic algorithm to perform inclusion checking on a RAM machine.
\end{abstract}

Key words:

PACS:

\section{Introduction}

XML schemas are an essential tool for the robustness of applications that involve XML data manipulation, transformation, integration, and, crucially,

Email addresses: dario.colazzo@lri.fr (Dario Colazzo), ghelli@di.unipi.it (Giorgio Ghelli), sartiani@di.unipi.it (Carlo Sartiani). 
data exchange. To solve any static analysis problem that involves such types one must first be able to reason about their inclusion and equivalence.

XML schema languages are designed to describe ordered data, but they usually offer some (limited) support to deal with cases where the order among some elements is not constrained. These "unordered" mechanisms bring the language out of the well-understood realm of tree-grammars and tree-automata, and have been subject to little foundational study, with the important exception of a recent work by Gelade, Martens, and Neven [7]. Here, the authors study a wide range of schema languages, and show that the addition of interleaving (also called shuffle) and counting operators raises the complexity of inclusion checking from PSPACE (or EXPTIME, for Extended DTDs) to EXPSPACE. These are completeness results, hence this is really bad news.

A previous result by Mayer and Stockmeyer [11] had already shown that the inclusion of Regular Expressions with interleaving alone is complete in EXPSPACE, hence showing that counting is not essential for the high cost. Gelade et al.'s paper [7] concludes with: "It would therefore be desirable to find robust subclasses for which the basic decision problems are in PTIME". Such subclasses could be used either to design a new schema language, or to design adaptive algorithms, that use the PTIME algorithm whenever is possible, and resort to the full algorithm when needed. To this aim, it is important that (i) the subclass covers large classes of XML types used in practice, (ii) it is easy to verify whether a schema belongs to the subclass.

Our Contribution In this paper we define a class of Regular Expressions (REs) with interleaving and numerical constraints whose inclusion can be checked in polynomial time. Our class is based on the following two restrictions: each expression is conflict-free (or single occurrence) meaning that no symbol appears twice, and Kleene star is only applied to symbols or to disjunctions of symbols. We use the name "conflict-free types" for this class of REs with interleaving and counting. These restrictions are severe, but, as shown in [4] and [5], they are actually met by the vast majority of the schemas that are used in practice. ${ }^{1}$ Polynomiality of inclusion of conflict-free types implies that XML types that use our conflict-free types as their content model can be checked for inclusion in PTIME as well; this result is well-known [7].

Our approach is based on the transformation of each type (i.e., each RE) into an equivalent set of constraints. Consider, for instance, the following type $T=(a[1 . .3] \cdot b[2 . .2])+c[1 . .2]$, and the following properties for a word $w$ in the language of $T$ :

(1) lower-bound: at least one of $a, b$, and $c$ appears in $w$;

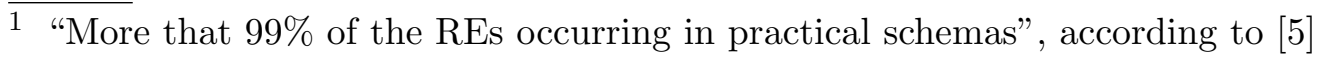


(2) cardinality: if $a$ is in $w$, it appears 1,2 or 3 times; if $b$ is there, it appears twice; if $c$ is there, it appears once or twice;

(3) upper-bound: no symbol out of $\{a, b, c\}$ is in $w$;

(4) exclusion: if one of $a, b$ is in $w$, then $c$ is not, and if $c$ is in $w$ then neither of $a, b$ is in $w$;

(5) co-occurrence: if $a$ is in $w$, then $b$ is in $w$, and vice versa; ${ }^{2}$

(6) order: no occurrence of $a$ may follow an occurrence of $b$.

It is easy to see that every $w$ in $T$ enjoys all of them. We will prove here that the opposite implication is true as well: every word that satisfies the six properties is indeed in $T$, i.e., that constraint set $(1)-(6)$ is complete for $T$.

We will generalize this observation, and will associate a complete set of constraints, in the six categories above, to any conflict-free type (we will actually encode exclusion constraints as order constraints); as a consequence, type inclusion will be reduced to constraint implication. We then prove that each class of constraints can be checked independently, and provide an algorithm to verify type inclusion $T<U$ in time $O\left(|T| *|U|+|U|^{2}\right)$, through constraint implication.

For those classes of regular expressions that are characterized by corresponding classes of automata, the complexity of non-emptiness of binary intersection is usually lower or equal to that of inclusion, since the first is typically reduced to automata intersection, while the second is reduced to automata complement plus intersection. We prove here that things go the opposite way with our conflict-free types, since we prove intersection to be NP-complete. While the membership algorithm we defined in [9] may be regarded as automata-based, we have not been able to find a really natural class of automata for conflict-free types. This result about intersection complexity seems to suggest that such automata may be subject to surprising properties.

The ability to transform a type into a complete set of constraints expressed in a limited variable-free logic is used here to design an efficient inclusion algorithm. We used the same characterization to define a linear-time membership algorithm for the same class of types in [9] (membership is NP-complete for REs with interleaving). We also believe that the constraints approach could be useful for other tasks, such as inclusion of general REs with interleaving into conflict-free types, or path containment under a DTD based on our type language, but we leave this for future work.

Complexity Model To study the complexity of our algorithms we base our analysis on the RAM (Random Access Machine) model. As usual, we assume

$\overline{2}$ The term co-occurrence constraint has an unrelated meaning in [1]; we use it as in $[12]$. 
that read/write operations on the RAM memory and on the input/output device are performed in unit time, as well as comparisons and arithmetic operations. Under these hypotheses, our algorithms, expressed in a Pascal-like pseudocode, can be simulated by RAM programs with the same asymptotic complexity.

Paper Outline The paper is structured as follows. Section 2 describes the data model, the type language, and the constraint language we are using. Section 3 shows how types can be characterized in terms of constraints, and proves both correctness and completeness of this characterization. Section 4, then, shows how the constraint characterization of Section 3 can be exploited to derive a correct and complete inclusion-checking algorithm. In Section 5 we show that intersection is NP-complete. In Sections 6 and 7, finally, we briefly revise some related works and draw our conclusions.

\section{Type Language and Constraint Language}

\subsection{The Type Language}

Gelade, Martens, and Neven showed that, if inclusion for a given class of regular expressions with interleaving and numerical constraints is in the complexity class $\mathcal{C}$, and $\mathcal{C}$ is closed under positive reductions (a property enjoyed by PTIME), then the complexity of inclusion for DTDs and single-type EDTDs that use the same class of regular expressions is in $\mathcal{C}$ too $[10,7]$. Hence, we can focus our study on a class of regular expression over strings, and our PTIME result will immediately imply the same complexity for the inclusion problem of the corresponding classes of DTDs and single-type EDTDs. Single-type EDTDs are the theoretical counterpart of XML Schema definitions (see [7]).

We adopt the usual definitions for string concatenation $w_{1} \cdot w_{2}$, and for the concatenation of two languages $L_{1} \cdot L_{2}$. The shuffle, or interleaving, operator $w_{1} \& w_{2}$ is also standard, and is defined as follows.

Definition $1\left(v \& w, L_{1} \& L_{2}\right)$ Given a finite alphabet $\Sigma$, the shuffle set of two words $v, w \in \Sigma^{*}$, or two languages $L_{1}, L_{2} \subseteq \Sigma^{*}$, is defined as follows; notice that each $v_{i}$ or $w_{i}$ may be the empty string $\epsilon$.

$$
\begin{gathered}
v \& w={ }_{\text {def }}\left\{v_{1} \cdot w_{1} \cdot \ldots \cdot v_{n} \cdot w_{n}\right. \\
\left.\mid v_{1} \cdot \ldots \cdot v_{n}=v, w_{1} \cdot \ldots \cdot w_{n}=w, v_{i} \in \Sigma^{*}, w_{i} \in \Sigma^{*}, n>0\right\} \\
L_{1} \& L_{2}={ }_{\text {def }} \bigcup_{w_{1} \in L_{1},}, w_{2} \in L_{2} \\
w_{1} \& w_{2}
\end{gathered}
$$


Example $2(a b) \&(X Y)$ contains the permutations of abXY where a comes before $b$ and $X$ comes before $Y$ :

$$
(a b) \&(X Y)=\{a b X Y, a X b Y, a X Y b, X a b Y, X a Y b, X Y a b\}
$$

When $v \in w_{1} \& w_{2}$, we say that $v$ is a shuffle of $w_{1}$ and $w_{2}$; for example, $w_{1} \cdot w_{2}$ and $w_{2} \cdot w_{1}$ are shuffles of $w_{1}$ and $w_{2}$.

We define $\mathbb{N}_{*}=\mathbb{N} \cup\{*\}$, and extend the standard order among naturals with $n \leq *$ for each $n \in \mathbb{N}_{*}$. We consider the following type language for strings over an alphabet $\Sigma$ :

$$
T::=\epsilon|a[m . . n]| T+T|T \cdot T| T \& T \mid T !
$$

where: $a \in \Sigma, m \in(\mathbb{N} \backslash\{0\}), n \in\left(\mathbb{N}_{*} \backslash\{0\}\right), n \geq m$, and, for any $T$ !, at least one of the subterms of $T$ has shape $a[m . . n]$.

Note that expressions like $a[0 . . n]$ are not allowed due to the condition on $m$; of course, the type $a[0 . . n]$ can be equivalently represented by $a[1 . . n]+\epsilon$. The type $T$ ! denotes $\llbracket T \rrbracket \backslash\{\epsilon\}$. The presence of an $a[m . . n]$ subterm in $T$ ! guarantees that $T$ contains at least one word that is different from $\epsilon$, hence $T$ ! is never empty, hence no type in our language is empty (Lemma 4).

Definition $3(S(w), S(T)$, Atoms $(T))$ For any string $w, S(w)$ is the set of all symbols appearing in $w$. For any type $T, \operatorname{Atoms}(T)$ is the set of all atoms $a[m . . n]$ appearing in $T$, and $S(T)$ is the set of all symbols appearing in $T$.

The semantics of types is defined as follows ( $|w|$ denotes the length of $w)$.

$$
\begin{aligned}
\llbracket \epsilon \rrbracket & =\{\epsilon\} \\
\llbracket a[m . . n \rrbracket \rrbracket & =\{w|S(w)=\{a\}, m \leq| w \mid \leq n\} \\
\llbracket T_{1}+T_{2} \rrbracket & =\llbracket T_{1} \rrbracket \cup \llbracket T_{2} \rrbracket \\
\llbracket T_{1} \cdot T_{2} \rrbracket & =\llbracket T_{1} \rrbracket \cdot \llbracket T_{2} \rrbracket \\
\llbracket T_{1} \& T_{2} \rrbracket & =\llbracket T_{1} \rrbracket \& \llbracket T_{2} \rrbracket \\
\llbracket T ! \rrbracket & =\llbracket T \rrbracket \backslash\{\epsilon\}
\end{aligned}
$$

The following Lemma can be easily proved by structural induction.

Lemma 4 (Not empty) For any type T:

$$
\begin{array}{r}
\llbracket T \rrbracket \neq \emptyset \\
a[m . . n] \in \operatorname{Atoms}(T) \Rightarrow \exists w \in \llbracket T \rrbracket . a \in S(w)
\end{array}
$$


It is worth noting that our type system lacks general repetition types $T^{*}$ or $T^{+}$. Repetition types are here generalized by counting, which, is, however, restricted to symbols, so that, for example, types like $(a \cdot b)^{*}$ cannot be expressed. However, it has been found that DTD and XSD (XML Schema Definition) schemas use repetition almost exclusively as $a^{\text {op }}$ or as $(a+\ldots+z)^{\text {op }}$ (see [5]), which can be immediately translated to our system. For instance, $(a+\ldots+z)^{*}$ can be expressed as $\left(a^{*} \& \ldots \& z^{*}\right)$, where $a^{*}$ is a shortcut for $\left.a[1 . . *]+\epsilon\right)$, while $(a+\ldots+z)^{+}$can be expressed as $\left(a^{*} \& \ldots \& z^{*}\right) !$

Restrictions on types, similar to those we consider here, have already been considered in some previous works. Bex et al. [4] studied simple expressions (introduced by Martens et al. in [10]), which are concatenation of factors $\left(a_{1}^{\mathrm{op}_{1}}+\ldots+a_{n}^{\mathrm{op}_{n}}\right)^{\mathrm{op}}$. Simple expressions can be trivially translated to our systems, and the authors measured that a $97 \%$ fraction of content models in XSD schemas consist of such expressions; however, they did not consider conflict-freedom. Chain Regular Expressions, defined in [5], is a similar class of expression, which satisfy conflict-freedom, hence all of them can be translated to our system, and the authors report measuring that a $99 \%$ of content models fall in this class.

We will use $\otimes$ to range over $\cdot$ and $\&$ when we need to specify common properties, such as, for example: $\llbracket T \otimes \epsilon \rrbracket=\llbracket \epsilon \otimes T \rrbracket=\llbracket T \rrbracket$. Some types contain the empty string $\epsilon$, and are characterized as follows $(\mathrm{N}(T)$ is read as " $T$ is nullable").

Definition $5 \mathrm{~N}(T)$ is a predicate on types, defined as follows:

$$
\begin{aligned}
\mathrm{N}(\epsilon) & =\text { true } \\
\mathrm{N}(a[m . . n]) & =\text { false } \\
\mathrm{N}(T !) & =\text { false } \\
\mathrm{N}\left(T+T^{\prime}\right) & =\mathrm{N}(T) \text { or } \mathrm{N}\left(T^{\prime}\right) \\
\mathrm{N}\left(T \otimes T^{\prime}\right) & =\mathrm{N}(T) \text { and } \mathrm{N}\left(T^{\prime}\right)
\end{aligned}
$$

The following Lemma is immediate by structural induction in the definition of $\mathrm{N}(T)$.

Lemma $6 \in \in \llbracket T \rrbracket$ iff $\mathrm{N}(T)$.

We can now define the notion of conflict-free types.

Definition 7 (Conflict-free types) A type $T$ is conflict-free iff for each subexpression $(U+V)$ or $(U \otimes V): S(U) \cap S(V)=\emptyset$.

Equivalently, a type $T$ is conflict-free if, for any two distinct subterms $a[m . . n]$ 
and $a^{\prime}\left[m^{\prime} . . n^{\prime}\right]$ that occur in $T, a$ is different from $a^{\prime}$.

Example 8 Consider the following type: $(a[1 . .1] \& b[1 . .1])+(a[1 . .1] \& c[1 . .1])$. This type generates the language $\{a b, b a, a c, c a\}$. This type is not conflict-free, since $S(a[1 . .1] \& b[1 . .1]) \cap S(a[1 . .1] \& c[1 . .1])=\{a\} \neq \emptyset$.

Consider now $a[1 . .1] \&(b[1 . .1]+c[1 . .1])$; it generates the same language, but is conflict-free since $a[1 . .1]$ and $(b[1 . .1]+c[1 . .1])$ have no common symbols.

Conflict-free DTDs have been considered many times before, because of their good properties and because of the high percentage of actual schemas that satisfy this constraint (see Section 6).

Hereafter, we will silently assume that every type is conflict-free.

\subsection{The Constraint Language}

We verify inclusion between $T$ and $U$ by translating them into constraint sets $C_{T}$ and $C_{U}$ and then by verifying that $C_{T}$ implies $C_{U}$. Constraints are expressed using the following logic, where $a, b \in \Sigma$ and $A, B \subseteq \Sigma, m \in(\mathbb{N} \backslash\{0\})$, $n \in\left(\mathbb{N}_{*} \backslash\{0\}\right)$, and $n \geq m$ :

$F::=A^{+}\left|A^{+} \Leftrightarrow B^{+}\right| a ?[m . . n]|\operatorname{upper}(A)| a \prec b\left|F \wedge F^{\prime}\right|$ true 
Satisfaction of a constraint $F$ by a word $w$, written $w \models F$, is defined as follows. ${ }^{3}$

$$
\begin{array}{lll}
w \models A^{+} & \Leftrightarrow & S(w) \cap A \neq \emptyset, \text { i.e. some } a \in A \text { appears in } w \\
w \models A^{+} \models B^{+} & \Leftrightarrow & w \models A^{+} \text {or } w \models B^{+} \\
w \models a ?[m . . n](n \neq *) & \Leftrightarrow & \text { if } a \text { appears in } w \text {, then it appears } \\
& & \text { at least } m \text { times and at most } n \text { times } \\
w \models a ?[m . . *] & \Leftrightarrow & \text { if } a \text { appears in } w \text {, then it appears } \\
& \quad \Leftrightarrow \quad S(w) \subseteq A \\
w \models \operatorname{upper}(A) & \Leftrightarrow \quad \text { there is no occurrence of } a \text { in } w \text { that follows } \\
w \models a \prec b & & \text { an occurrence of } b \text { in } w
\end{array}
$$

The following special cases of the above definition are worth noticing.

$$
\begin{aligned}
& \epsilon \not \models A^{+} \quad \epsilon \models \operatorname{upper}(A) \quad \epsilon \models a ?[m . . n] \\
& \epsilon \models a \prec b \quad b \models a \prec b \quad a b a \not \models a \prec b \\
& w \not \models \emptyset^{+} \quad w \models \emptyset^{+} \Leftrightarrow A^{+} \quad w \models \emptyset^{+} \Leftrightarrow \emptyset^{+}
\end{aligned}
$$

Observe that $A^{+}$is monotone, i.e., $w \models A^{+}$and $w$ is a subword of $w^{\prime}$ imply that $w^{\prime} \models A^{+}$, while upper $(A)$ and $a \prec b$ are anti-monotone.

We use the following abbreviations:

$$
\begin{aligned}
a^{+} & =\text {def } \quad\{a\}^{+} \\
a \prec \succ b & =\operatorname{def}_{\text {ef }} \quad(a \prec b) \wedge(b \prec a) \\
A \prec B & =\bigwedge_{\text {def }} \quad \bigwedge_{a \in A, b \in B} a \prec b \\
A \prec \succ B & ={ }_{\operatorname{def}} \quad \bigwedge_{a \in A, b \in B} a \prec \succ b
\end{aligned}
$$

The next propositions specify that $A \prec \succ B$ encodes mutual exclusion between sets of symbols.

3 Notice that $A^{+} \Leftrightarrow b^{+}$differs from the sibling constraint $A \Downarrow b$ of [13], since $A^{+} \Leftrightarrow b^{+}$means "if one symbol of $A$ is in $w$ then $b$ is in $w$ ", while $A \Downarrow b$ means "if all symbols of $A$ are in $w$ then $b$ is in $w "$. 
Proposition $9 w \models a \prec \succ b \Leftrightarrow a$ and $b$ are not both in $S(w)$

Proposition $10 w \models A \prec \succ B \Leftrightarrow w \not \models A^{+} \wedge B^{+}$

Proof. By Proposition 9, we observe that $w \models A \prec \succ B$ holds if and only if, for each $a \in A, b \in B,\{a, b\} \nsubseteq S(w)$. This means that either $A \cap S(w)=\emptyset$ or $B \cap S(w)=\emptyset$, that is, $w \not \models A^{+} \wedge B^{+}$.

We extend the $S\left(_{-}\right)$notation to formulas.

Definition $11 a \in S(F)$ if one of the following is a subterm of $F: a ?[m . . n]$, $a \prec b, A^{+}, A^{+} \Leftrightarrow B^{+}$, upper $(A)$, where, in the last three cases, $a \in A$ or $a \in B$.

The atomic operators are all mutually independent: only $A^{+}$can force the presence of a symbol independently of any other, only $A^{+} \Leftrightarrow B^{+}$induces a positive correlation between the presence of two symbols, only $a$ ? $[m . . n]$ can count, only upper $(A)$ is affected by the presence of a symbol that is not in $S(F)$, and only $a \prec b$ is affected by order. However, combinations of the atomic operators can be mutually related (see Proposition 10, for example).

\section{Characterization of Types as Constraints}

\subsection{Constraint Extraction}

We first extend satisfaction from words to types, as follows.

Definition $12 T \models F \Leftrightarrow \forall w \in \llbracket T \rrbracket . w \models F$

We associate to each type $T$ a formula $S^{+}(T)$ that tests for the presence of one of its symbols, as follows.

Definition $13 S^{+}(T)=(S(T))^{+}$

We also define $I f_{T}(F)$, which denotes either the formula $F$, or the formula true, depending on whether $\mathrm{N}(T)$ holds. We use it to express the lower-bound and co-occurrence constraints, since they only hold for non-nullable types.

Definition 14 (If-non-nullable) If ${ }_{T}(F)$ denotes the formula true if $\mathrm{N}(T)$ holds, and denotes the formula $F$ otherwise.

We can now endow a type $T$ with five sets of constraints. We divide them in "nested constraints" (co-occurrence, order-and-exclusion), whose definition 
depends on the nesting structures of the type, and "flat constraints" (lowerbound, cardinality, and upper-bound), whose definition only depends on the leaves of the types, and on its nullability.

\section{Definition 15 (Flat constraints)}

$$
\text { Lower-bound: } \quad \operatorname{SIf}(T)={ }_{\operatorname{def}} \operatorname{If}_{T}\left(S^{+}(T)\right)
$$

Cardinality: ZeroMinMax $(T)={ }_{\operatorname{def}} \bigwedge_{a[m . . n] \in \operatorname{Atoms}(T)} a ?[m . . n]$

Upper-bound: $\quad \operatorname{upperS}(T)={ }_{\text {def }} \operatorname{upper}(S(T))$

Flat constraints: $\quad \mathcal{F C}(T)={ }_{\text {def }} \operatorname{SIf}(T) \wedge \operatorname{ZeroMinMax}(T) \wedge \operatorname{upperS}(T)$

Nested constraints are defined by induction over the type structure.

\section{Definition 16 (Nested constraints)}

Co-occurrence:

$$
\begin{array}{lcl}
\mathcal{C C}\left(T_{1}+T_{2}\right) & ={ }_{\text {def }} & \mathcal{C C}\left(T_{1}\right) \wedge \mathcal{C C}\left(T_{2}\right) \\
& ={ }_{\text {def }} & I_{T_{2}}\left(S^{+}\left(T_{1}\right) \mapsto S^{+}\left(T_{2}\right)\right) \\
& & \wedge I f_{T_{1}}\left(S^{+}\left(T_{2}\right) \mapsto S^{+}\left(T_{1}\right)\right) \\
& \wedge \mathcal{C C}\left(T_{1}\right) \wedge \mathcal{C C}\left(T_{2}\right) \\
\mathcal{C C}(T !) & ={ }_{\text {def }} \mathcal{C C}(T) \\
\mathcal{C C}(\epsilon)={ }_{\text {def }} \mathcal{C C}(a[m . . n])= & { }_{\text {def }} \text { true }
\end{array}
$$

Order and exclusion:

$$
\begin{array}{ll}
\mathcal{O C}\left(T_{1}+T_{2}\right) & ={ }_{\text {def }}\left(S\left(T_{1}\right) \prec \succ S\left(T_{2}\right)\right) \wedge \mathcal{O C}\left(T_{1}\right) \wedge \mathcal{O C}\left(T_{2}\right) \\
\mathcal{O C}\left(T_{1} \& T_{2}\right) & ={ }_{\text {def }} \mathcal{O C}\left(T_{1}\right) \wedge \mathcal{O C}\left(T_{2}\right) \\
\mathcal{O C}\left(T_{1} \cdot T_{2}\right) & { }_{\text {def }}\left(S\left(T_{1}\right) \prec S\left(T_{2}\right)\right) \wedge \mathcal{O C}\left(T_{1}\right) \wedge \mathcal{O C}\left(T_{2}\right) \\
\mathcal{O C}(T !) & ={ }_{\text {def }} \mathcal{O C}(T) \\
\mathcal{O C}(\epsilon)={ }_{\text {def }} \mathcal{O C}(a[m . . n]) & ={ }_{\text {def }} \text { true }
\end{array}
$$

Nested constraints:

$$
\mathcal{N C}(T) \quad={ }_{\operatorname{def}} \mathcal{C C}(T) \wedge \mathcal{O C}(T)
$$

As a consequence of the above definition, nested constraints have the following property. 


\section{Proposition $17(\mathcal{N C}(T))$}

$$
\begin{aligned}
\mathcal{N C}\left(T_{1}+T_{2}\right)= & \left(S\left(T_{1}\right) \prec \succ S\left(T_{2}\right)\right) \wedge \mathcal{N C}\left(T_{1}\right) \wedge \mathcal{N C}\left(T_{2}\right) \\
\mathcal{N C}\left(T_{1} \& T_{2}\right)= & \left(I f_{T_{2}}\left(S^{+}\left(T_{1}\right) \boxminus S^{+}\left(T_{2}\right)\right) \wedge\left(I f_{T_{1}}\left(S^{+}\left(T_{2}\right) \models S^{+}\left(T_{1}\right)\right)\right) \wedge\right. \\
& \mathcal{N C}\left(T_{1}\right) \wedge \mathcal{N C}\left(T_{2}\right) \\
\mathcal{N C}\left(T_{1} \cdot T_{2}\right)= & \left(S\left(T_{1}\right) \prec S\left(T_{2}\right)\right) \\
& \wedge\left(I f_{T_{2}}\left(S^{+}\left(T_{1}\right) \boxminus S^{+}\left(T_{2}\right)\right)\right) \wedge\left(I f_{T_{1}}\left(S^{+}\left(T_{2}\right) \mapsto S^{+}\left(T_{1}\right)\right)\right) \\
& \wedge \mathcal{N C}\left(T_{1}\right) \wedge \mathcal{N C}\left(T_{2}\right) \\
\mathcal{N C}(T !)= & \mathcal{N C}(T) \\
\mathcal{N C}(\epsilon)= & \text { true } \\
\mathcal{N C}(a[m . . n])= & \text { true }
\end{aligned}
$$

\subsection{Correctness and Completeness of Constraints}

We plan to prove the following theorem, that specifies that the constraint system completely captures the semantics of conflict-free types.

Theorem 18 Given a conflict-free type $T$, it holds that:

$$
w \in \llbracket T \rrbracket \Leftrightarrow w \models \mathcal{F C}(T) \wedge \mathcal{N C}(T)
$$

We first prove that constraints are complete, i.e., whenever $w$ satisfies all the five groups of constraints associated with $T$, then $w \in \llbracket T \rrbracket$.

Proposition $19(\operatorname{ZeroMinMax}(T))$

$$
\begin{aligned}
& w \models \operatorname{ZeroMinMax}\left(T_{1}+T_{2}\right) \Rightarrow w \models \operatorname{ZeroMinMax}\left(T_{1}\right) \wedge \operatorname{ZeroMinMax}\left(T_{2}\right) \\
& w \models \operatorname{ZeroMinMax}\left(T_{1} \otimes T_{2}\right) \Rightarrow w \models \operatorname{ZeroMinMax}\left(T_{1}\right) \wedge \operatorname{ZeroMinMax}\left(T_{2}\right)
\end{aligned}
$$

Proof. By definition of ZeroMinMax $(T)$.

Definition 20 (Word projection) We define the projection of a word $w$ onto $A\left(\left.w\right|_{A}\right)$ as the string obtained from $w$ by removing all the symbols that are not in $A$.

\section{Proposition 21}

$$
w \models S^{+}\left(T_{1}\right) \wedge w \models I f_{T_{2}}\left(S^{+}\left(T_{1}\right) \Leftrightarrow S^{+}\left(T_{2}\right)\right) \Rightarrow w \models \operatorname{SIf}\left(T_{2}\right)
$$

We can now prove the crucial completeness theorem. 


\section{Theorem 22 (Completeness of constraints)}

$$
w \models(\mathcal{F C}(T) \wedge \mathcal{N C}(T)) \quad \Rightarrow \quad w \in \llbracket T \rrbracket
$$

\section{Proof.}

For the sake of convenience, we will use ZMM-SIf $(T)$ as a shortcut for ZeroMinMax $(T) \wedge$ $\operatorname{SIf}(T)$, so that we can rewrite the thesis as

$$
w \models(\operatorname{upperS}(T) \wedge \mathrm{ZMM-SIf}(T) \wedge \mathcal{N C}(T)) \Rightarrow w \in \llbracket T \rrbracket
$$

We prove the following fact, by case inspection and structural induction on $T$.

$$
\left.w \models(Z M M-S I f(T) \wedge \mathcal{N C}(T)) \Rightarrow w\right|_{S(T)} \in \llbracket T \rrbracket
$$

The theorem follows because $w \models \operatorname{upperS}(T)$ implies that $w=\left.w\right|_{S(T)}$.

We first observe that $\left.w\right|_{S(T)}=\epsilon$ and $w=S I f(T)$ imply the thesis $\left.w\right|_{S(T)} \in \llbracket T \rrbracket$. Indeed, $\left.w\right|_{S(T)}=\epsilon$ implies that $w \not \models S^{+}(T)$, hence, the hypothesis $w \models S I f(T)$ implies that $\mathrm{N}(T)$ is true, which in turn implies that $\epsilon \in \llbracket T \rrbracket$, i.e. $\left.w\right|_{S(T)} \in \llbracket T \rrbracket$.

Having dealt with the $\left.w\right|_{S(T)}=\epsilon$ case, in the following we assume that $\left.w\right|_{S(T)}=$ $a_{1} \cdot \ldots \cdot a_{n}$, where $n \neq 0$ (where, for each $i$ and $j$, the symbol $a_{i}$ may be either equal or different from $a_{j}$ ).

$\mathbf{T}=\epsilon:$

Trivial, as $\left.w\right|_{S(\epsilon)}=\epsilon$ and $\epsilon \in \llbracket \epsilon \rrbracket$.

$\mathbf{T}=\mathbf{a}[\mathbf{m} . . \mathbf{n}]:$

Since $\mathrm{N}(T)$ is false, $w \models$ ZMM-SIf $(T)$ implies that $w \models \operatorname{ZeroMinMax}(T) \wedge$ $S^{+}(T)$, i.e., $w \models \operatorname{ZeroMinMax}(a[m . . n]) \wedge a^{+}$, i.e., $w \models a$ ? $[m . . n] \wedge a^{+}$, hence $\left.w\right|_{S(a[m . n])} \in \llbracket a[m . . n] \rrbracket$.

$\mathbf{T}=\mathbf{T}_{1}+\mathbf{T}_{2}$

Let $\left.w\right|_{S(T)}=a_{1} \ldots a_{n}$, and assume, without loss of generality, that $a_{1} \in S\left(T_{1}\right)$.

By hypothesis and Proposition 17 we have that $w \models \operatorname{ZMM-SIf}\left(T_{1}+T_{2}\right) \wedge$ $\left(S\left(T_{1}\right) \prec \succ S\left(T_{2}\right)\right) \wedge \mathcal{N C}\left(T_{1}\right) \wedge \mathcal{N C}\left(T_{2}\right)$. As $\left.w\right|_{S(T)}=a_{1} \cdot \ldots \cdot a_{n}$ with $a_{1} \in S\left(T_{1}\right)$, we also have that $w \models S^{+}\left(T_{1}\right)$.

This implies that $w \models S I f\left(T_{1}\right)$ (by definition of $\left.\operatorname{SIf}()\right)$ and that $w \not \models S^{+}\left(T_{2}\right)$ (by Proposition 10). This, in turn, implies $\left.w\right|_{S\left(T_{1}+T_{2}\right)}=\left.w\right|_{S\left(T_{1}\right)}(*)$. By Proposi- 
tion 19 and by $w \models Z M M-S I f\left(T_{1}+T_{2}\right)$ we obtain that $w \models \operatorname{ZeroMinMax}\left(T_{1}\right)$. Putting all together, $w \models$ ZMM-SIf $\left(T_{1}\right) \wedge \mathcal{N C}\left(T_{1}\right)$.

By induction we have that $\left.w\right|_{S\left(T_{1}\right)} \in \llbracket T_{1} \rrbracket$; hence, by $(*)$, we get $\left.w\right|_{S\left(T_{1}+T_{2}\right)} \in$ $\llbracket T_{1} \rrbracket$, which, in turn, implies that $\left.w\right|_{S\left(T_{1}+T_{2}\right)} \in \llbracket T_{1}+T_{2} \rrbracket$.

$\mathrm{T}=\mathrm{T}_{1} \cdot \mathrm{T}_{2}$

We have two possible cases:

(1) $\left.w\right|_{S(T)}=a_{1} \cdot \ldots \cdot a_{n}$ and $a_{1} \in S\left(T_{1}\right)$;

(2) $\left.w\right|_{S(T)}=a_{1} \cdot \ldots \cdot a_{n}$ and $a_{1} \in S\left(T_{2}\right)$.

In both cases, by hypothesis and Proposition 17 we have that $\left(^{*}\right)$ :

$$
\begin{aligned}
w \models \text { ZMM-SIf }\left(T_{1} \cdot T_{2}\right) & \wedge\left(I_{T_{2}}\left(S^{+}\left(T_{1}\right) \models S^{+}\left(T_{2}\right)\right)\right) \\
& \wedge\left(I f_{T_{1}}\left(S^{+}\left(T_{2}\right) \models S^{+}\left(T_{1}\right)\right)\right) \\
& \wedge\left(S\left(T_{1}\right) \prec S\left(T_{2}\right)\right) \\
& \wedge \mathcal{N C}\left(T_{1}\right) \wedge \mathcal{N C}\left(T_{2}\right)
\end{aligned}
$$

Case $1\left(\left.w\right|_{S(T)}=a_{1} \cdot \ldots \cdot a_{n}\right.$ and $\left.a_{1} \in S\left(T_{1}\right)\right)$.

By $\left({ }^{*}\right)$, since $\left.w\right|_{S(T)}=a_{1} \cdot \ldots \cdot a_{n}$ and $a_{1} \in S\left(T_{1}\right)$, we have that $w \models S^{+}\left(T_{1}\right)$, which implies that $w \models \operatorname{SIf}\left(T_{1}\right)$ (by definition of $\left.\operatorname{SIf}()\right)$ and that $w \models \operatorname{SIf}\left(T_{2}\right)$ (by hypothesis and by Proposition 21). By Proposition 19 we conclude that $w \models \mathrm{ZMM}-\operatorname{SIf}\left(T_{1}\right) \wedge \mathrm{ZMM}-\mathrm{SIf}\left(T_{2}\right)$.

Let us define $w_{1}=\left.w\right|_{S\left(T_{1}\right)}$ and $w_{2}=\left.w\right|_{S\left(T_{2}\right)}$. As $w \models \mathcal{N C}\left(T_{1}\right) \wedge \mathcal{N C}\left(T_{2}\right)$, by induction we obtain that $w_{1} \in \llbracket T_{1} \rrbracket$ and $w_{2} \in \llbracket T_{2} \rrbracket$.

By conflict-freedom, $w_{1}$ and $w_{2}$ do not contain any common symbols, hence, from the constraint $S\left(T_{1}\right) \prec S\left(T_{2}\right)$ we obtain that each symbol of $w_{1}$ precedes each symbol of $w_{2}$ in $w$. As a consequence, $\left.w\right|_{S\left(T_{1} \cdot T_{2}\right)}=\left.\left.w\right|_{S\left(T_{1}\right)} \cdot w\right|_{S\left(T_{2}\right)}=w_{1} \cdot w_{2}$. Thus, $\left.w\right|_{S\left(T_{1} \cdot T_{2}\right)} \in \llbracket T_{1} \cdot T_{2} \rrbracket$.

Case $2\left(\left.w\right|_{S(T)}=a_{1} \cdot \ldots \cdot a_{n}\right.$ and $\left.a_{1} \in S\left(T_{2}\right)\right)$.

By $\left(^{*}\right)$, since $\left.w\right|_{S(T)}=a_{1} \cdot \ldots \cdot a_{n}$ and $a_{1} \in S\left(T_{2}\right)$, we obtain that $w \models S^{+}\left(T_{2}\right)$, which implies that $w \models S I f\left(T_{1}\right)$ (by Proposition 21) and that $w \models S I f\left(T_{2}\right)$ (by definition). By Proposition 19 we conclude that $w \models \operatorname{ZMM-SIf}\left(T_{1}\right) \wedge$ ZMM-SIf $\left(T_{2}\right)$. As $w \models \mathcal{N C}\left(T_{1}\right) \wedge \mathcal{N C}\left(T_{2}\right)$, by induction we obtain that $\left.w\right|_{S\left(T_{1}\right)} \in$ $\llbracket T_{1} \rrbracket$ and $\left.w\right|_{S\left(T_{2}\right)} \in \llbracket T_{2} \rrbracket$. 
$w \models\left(S\left(T_{1}\right) \prec S\left(T_{2}\right)\right)$ and $a_{1} \in S\left(T_{2}\right)$ imply that $w \not \models S^{+}\left(T_{1}\right)$, i.e., $\left.w\right|_{S\left(T_{1}\right)}=\epsilon$. Hence, $\left.w\right|_{S\left(T_{1} \cdot T_{2}\right)}=\left.w\right|_{S\left(T_{2}\right)}=\left.\epsilon \cdot w\right|_{S\left(T_{2}\right)}=\left.\left.w\right|_{S\left(T_{1}\right)} \cdot w\right|_{S\left(T_{2}\right)}$. Hence, by $\left.w\right|_{S\left(T_{1}\right)} \in$ $\llbracket T_{1} \rrbracket$ and $\left.w\right|_{S\left(T_{2}\right)} \in \llbracket T_{2} \rrbracket$, we conclude that $\left.w\right|_{S\left(T_{1} \cdot T_{2}\right)} \in \llbracket T_{1} \cdot T_{2} \rrbracket$.

$\mathbf{T}=\mathbf{T}_{1} \& \mathbf{T}_{2}$

Let $\left.w\right|_{S(T)}=a_{1} \cdot \ldots a_{n}$ and assume, without loss of generality, that $a_{1} \in S\left(T_{1}\right)$.

By hypothesis and Proposition 17 we have that:

$$
\begin{aligned}
w \models \operatorname{ZMM}-S I f\left(T_{1} \& T_{2}\right) & \wedge\left(I_{T_{2}}\left(S^{+}\left(T_{1}\right) \boxminus S^{+}\left(T_{2}\right)\right)\right) \\
& \wedge\left(I_{T_{1}}\left(S^{+}\left(T_{2}\right) \boxminus S^{+}\left(T_{1}\right)\right)\right) \\
& \wedge \mathcal{N C}\left(T_{1}\right) \wedge \mathcal{N C}\left(T_{2}\right)
\end{aligned}
$$

Since $\left.w\right|_{S(T)}=a_{1} \cdot \ldots \cdot a_{n}$, we have that $w \models S^{+}\left(T_{1}\right)$, from which we obtain that $w \models S I f\left(T_{1}\right)$ (by definition) and $w \models S I f\left(T_{2}\right)$.

By Proposition 19 it follows that $w \models \mathrm{ZMM-SIf}\left(T_{1}\right) \wedge \mathrm{ZMM-SIf}\left(T_{2}\right)$.

As $w \models \mathcal{N C}\left(T_{1}\right) \wedge \mathcal{N C}\left(T_{2}\right)$, by induction we obtain that $w_{1}=\left.w\right|_{S\left(T_{1}\right)} \in \llbracket T_{1} \rrbracket$ and that $w_{2}=\left.w\right|_{S\left(T_{2}\right)} \in \llbracket T_{2} \rrbracket$.

By the conflict freedom hypothesis, $S\left(T_{1}\right) \cap S\left(T_{2}\right)=\emptyset$, hence $w$ is a shuffle of $w_{1} \cdot w_{2} \cdot w_{3}$, where the symbols in $w_{3}$ are not present in $S\left(T_{1} \& T_{2}\right)$. As a consequence, $\left.w\right|_{S\left(T_{1} \& T_{2}\right)} \in w_{1} \& w_{2}$, which implies that $\left.w\right|_{S\left(T_{1} \& T_{2}\right)} \in \llbracket T_{1} \& T_{2} \rrbracket$.

$\mathbf{T}=\mathbf{T}_{\mathbf{1}}$ ! : By the hypothesis $w \models S I f(T)$ and by the fact that $T$ is not nullable we deduce $w \neq \epsilon$ and $w \models S^{+}\left(T_{1}\right.$ !), hence $w \models S^{+}\left(T_{1}\right)$. From $w \models \mathcal{N C}\left(T_{1}\right.$ !) and $w \models \operatorname{ZeroMinMax}\left(T_{1}\right.$ !) we get $w \models \mathcal{N C}\left(T_{1}\right)$ and $w \models \operatorname{ZeroMinMax}\left(T_{1}\right)$. Hence, by induction, $\left.w\right|_{S\left(T_{1}\right)} \in \llbracket T_{1} \rrbracket$, and $\left.w\right|_{S\left(T_{1} !\right)} \in \llbracket T_{1} ! \rrbracket$ follows from $w \neq$ $\epsilon$.

In order to prove soundness, we use the following lemma specifying that the value of any formula $F$ over $w$ does not change if any letter $a$ that is not in $S(F)$ is added or deleted from $w$, provided that $F$ does not contain the upper $(A)$ operator. Recall that upper $(A)$ is only used to express upper-bound constraints.

Lemma 23 (Irrelevance) Assume that upper $(A)$ does not appear in $F$, for any $A$. Then, for any $B \supseteq S(F)$, and for any $w$ :

$$
\left.w \models F \Leftrightarrow w\right|_{B} \models F
$$




\section{Proof.}

By induction on the structure of $F$, and by case analysis.

$F=A^{+}: w \models A^{+}$iff $S(w) \cap A \neq \emptyset$ : this condition is not affected by projecting a word onto $B \supseteq A$, hence $\left.w \models F \Leftrightarrow w\right|_{B} \models F$.

$F=A_{1}{ }^{+} \Leftrightarrow A_{2}{ }^{+}: w \models A_{1}{ }^{+} \Leftrightarrow A_{2}{ }^{+}$iff either $S(w) \cap A_{1}=\emptyset$ or $S(w) \cap A_{2} \neq \emptyset$ : this condition is not affected by projecting a word onto $B \supseteq\left(A_{1} \cup A_{2}\right)$.

$F=a \prec b$ : $w \models a \prec b$ iff there is no occurrence of $a$ in $w$ that precedes one occurrence of $b$ in $w$; this is not affected by projecting $w$ onto $B \supseteq\{a, b\}$.

$F=a ?[m . . n]: w \models a ?[m . . n]$ iff, if $a$ appears in $w$, then it appears at least $m$ times and at most $n$ times: this is not affected by projecting $w$ onto $B \supseteq\{a\}$.

$F=F_{1} \wedge F_{2}$ : let $B \supseteq S(F)$; then, $B \supseteq S\left(F_{1}\right)$ and $B \supseteq S\left(F_{2}\right)$, hence the thesis follows by induction.

$F=$ true: trivial.

The soundness of our constraints is stated by Theorem 24 .

\section{Theorem 24 (Soundness)}

$$
w \in \llbracket T \rrbracket \Rightarrow w \models \mathcal{F C}(T) \wedge \mathcal{N C}(T)
$$

\section{Proof.}

We first prove $w \in \llbracket T \rrbracket \Rightarrow w \models \mathcal{F C}(T)$.

The implication $w \in \llbracket T \rrbracket \Rightarrow w \models \operatorname{upperS}(T)$ is immediate by structural induction on the definition of types.

For $w \in \llbracket T \rrbracket \Rightarrow w \models S I f(T)$, we have two cases. If $w=\epsilon$, then $w \in \llbracket T \rrbracket$ implies that $\mathrm{N}(T)$, and therefore $\operatorname{SIf}(T)=$ true, hence $w \models S I f(T)$. If $w \neq \epsilon$, then $S(w) \neq \emptyset$, hence, by $w \models \operatorname{upperS}(T)$, $w$ contains one symbol of $S(T)$, hence $w \models S I f(T)$.

For $w \in \llbracket T \rrbracket \Rightarrow w \models \operatorname{ZeroMinMax}(T)$, we proceed by case inspection and induction on $T$.

$\mathbf{T}=\epsilon: w \in \llbracket T \rrbracket$ implies $w=\epsilon$, and, by definition, $\epsilon \models \operatorname{ZeroMinMax}(T)$.

$\mathbf{T}=\mathbf{a}[\mathbf{m} . . \mathbf{n}]:$ Immediate 
$\mathbf{T}=\mathbf{T}_{\mathbf{1}}+\mathbf{T}_{\mathbf{2}}$ : Consider $w \in \llbracket T \rrbracket$, and assume, without loss of generality, that $w \in \llbracket T_{1} \rrbracket$. By induction we have $w \models \operatorname{ZeroMinMax}\left(T_{1}\right)$. By $S\left(T_{1}\right) \cap S\left(T_{2}\right)=\emptyset$ and $w \models \operatorname{upperS}\left(T_{1}\right)$, we also have that, for any $a[m . . n] \in \operatorname{Atoms}\left(T_{2}\right), a \notin$ $S(w)$, hence $w \models a ?[m . . n]$.

$\mathbf{T}=\mathbf{T}_{\mathbf{1}} \otimes \mathbf{T}_{\mathbf{2}}$ : Consider $w \in \llbracket T_{1} \otimes T_{2} \rrbracket ;$ by definition, there exist $w_{1} \in \llbracket T_{1} \rrbracket$ and $w_{2} \in \llbracket T_{2} \rrbracket$ such that $w \in w_{1} \& w_{2}$. By induction, $w_{1} \models \operatorname{ZeroMinMax}\left(T_{1}\right)$, i.e., for any $a[m . . n] \in \operatorname{Atoms}\left(T_{1}\right) w_{1} \models a ?[m . . n]$. From $S\left(T_{1}\right) \cap S\left(T_{2}\right)=\emptyset$ and $w_{2}=$ upperS $\left(T_{2}\right)$, we deduce that $\left.w\right|_{S\left(T_{1}\right)}=w_{1}$, hence, for any $a[m . n] \in \operatorname{Atoms}\left(T_{1}\right)$, $w \models a ?[m . n]$. In the same way, we prove that, for any $a[m . . n] \in \operatorname{Atoms}\left(T_{2}\right)$, $w \models a ?[m . . n]$, hence $w \models \operatorname{ZeroMinMax}(T)$.

$\mathbf{T}=\mathbf{T}_{\mathbf{1}}$ !: If $w \in \llbracket T \rrbracket$, then $w \in \llbracket T_{1} \rrbracket$ and $w \neq \epsilon$. By induction, $w \models \operatorname{ZeroMinMax}\left(T_{1}\right)$, hence $w \models \operatorname{ZeroMinMax}(T)$.

We now prove $w \in \llbracket T \rrbracket \Rightarrow w \models \mathcal{N C}(T)$.

We first observe that, for each $T, \epsilon=\mathcal{N C}(T)$, because $\epsilon$ trivially satisfies any order constraint, and it also satisfies any non-trivial co-occurrence constraint If $T_{2}\left(S^{+}\left(T_{1}\right) \Leftrightarrow S^{+}\left(T_{2}\right)\right)$ by falsifying the hypothesis $S^{+}\left(T_{1}\right)$. This observation will be crucial in the $T_{1}+T_{2}$ case.

We now proceed by case inspection and induction on $T$. The cases for $\epsilon$ and $a[m . . n]$ are trivial.

$\mathbf{T}=\mathbf{T}_{\mathbf{1}} \& \mathbf{T}_{\mathbf{2}}: \mathcal{N C}\left(T_{1} \& T_{2}\right)$ is defined as follows

$$
\left(I f_{T_{2}}\left(S^{+}\left(T_{1}\right) \boxminus S^{+}\left(T_{2}\right)\right)\right) \wedge\left(I f_{T_{1}}\left(S^{+}\left(T_{2}\right) \boxminus S^{+}\left(T_{1}\right)\right)\right) \wedge \mathcal{N C}\left(T_{1}\right) \wedge \mathcal{N C}\left(T_{2}\right)
$$

Consider $w \in \llbracket T \rrbracket$. We observe that, since $S\left(T_{1}\right) \cap S\left(T_{2}\right)=\emptyset$, we have $\left.w\right|_{S\left(T_{1}\right)} \in \llbracket T_{1} \rrbracket$ and $\left.w\right|_{S\left(T_{2}\right)} \in \llbracket T_{2} \rrbracket$, and therefore $\left.w\right|_{S\left(T_{1}\right)} \models \mathcal{N C}\left(T_{1}\right)$ and $\left.w\right|_{S\left(T_{2}\right)} \models \mathcal{N C}\left(T_{2}\right)$, by induction. Since $S\left(\mathcal{N C}\left(T_{i}\right)\right) \subseteq S\left(T_{i}\right)$, we have that $\left.w\right|_{S\left(T_{i}\right)} \models \mathcal{N C}\left(T_{i}\right)$ implies $w \models \mathcal{N C}\left(T_{i}\right)$, for $i=1,2$, by Lemma 23. Similarly, $\left.w\right|_{S\left(T_{2}\right)} \models S I f\left(T_{2}\right)$, which holds by correctness of flat constraints, implies that $w \models S I f\left(T_{2}\right)$, hence that $w \models I f_{T_{2}}\left(S^{+}\left(T_{1}\right) \Leftrightarrow S^{+}\left(T_{2}\right)\right)$. The constraint $w \models I f_{T_{1}}\left(S^{+}\left(T_{2}\right) \Leftrightarrow S^{+}\left(T_{1}\right)\right)$ is similar.

$\mathbf{T}=\mathbf{T}_{\mathbf{1}} \cdot \mathbf{T}_{\mathbf{2}}$ : This is similar to the previous case, but we also have to prove that $w \models S\left(T_{1}\right) \prec S\left(T_{2}\right)$. Assume $w \in \llbracket T \rrbracket$; then, there exist $w_{1} \in \llbracket T_{1} \rrbracket$ and $w_{2} \in \llbracket T_{2} \rrbracket$ such that $w=w_{1} \cdot w_{2}$. Let $a_{1} \in S\left(T_{1}\right)$ and $a_{2} \in S\left(T_{2}\right)$; we must prove that no occurrence of $a_{1}$ is present in $w$ after an occurrence of $a_{2}$. This follows immediately from the fact that every occurrence of $a_{1}$ is in $w_{1}$ and every occurrence of $a_{2}$ is in $w_{2}$, because of $w_{i} \models \operatorname{upperS}\left(T_{i}\right)$, and because of $S\left(T_{1}\right) \cap S\left(T_{2}\right)=\emptyset$. 
$\mathbf{T}=\mathbf{T}_{1}+\mathbf{T}_{\mathbf{2}}:$ We have:

$$
\mathcal{N C}\left(T_{1}+T_{2}\right)=\left(S\left(T_{1}\right) \prec \succ S\left(T_{2}\right)\right) \wedge \mathcal{N C}\left(T_{1}\right) \wedge \mathcal{N C}\left(T_{2}\right)
$$

Consider $w \in \llbracket T_{1}+T_{2} \rrbracket$. W.l.o.g., we can assume $w \in \llbracket T_{1} \rrbracket$. By induction we have $w=\mathcal{N C}\left(T_{1}\right)$. Moreover, from $S\left(T_{1}\right) \cap S\left(T_{2}\right)=\emptyset$, we have that $\left.w\right|_{S\left(T_{2}\right)}=\epsilon$, hence $\left.w\right|_{S\left(T_{2}\right)}=\mathcal{N C}\left(T_{2}\right)$, hence, by Lemma $23, w \models \mathcal{N C}\left(T_{2}\right)$.

To derive $w \models S\left(T_{1}\right) \prec \succ S\left(T_{2}\right)$, we observe that by $w \in \llbracket T_{1}+T_{2} \rrbracket$ and $S\left(T_{1}\right) \cap S\left(T_{2}\right)=\emptyset$ we have $w \not \models S\left(T_{1}\right)^{+} \wedge S\left(T_{2}\right)^{+}$. Therefore, by Proposition 10 we obtain $w \models S\left(T_{1}\right) \prec \succ S\left(T_{2}\right)$.

$\mathbf{T}=\mathbf{T}_{\mathbf{1}}$ !: We have $\llbracket T \rrbracket \subseteq \llbracket T_{1} \rrbracket$, hence $w \in \llbracket T_{1} \rrbracket$, hence, by induction, $w \models$ $\mathcal{N C}\left(T_{1}\right)$, hence $w \models \mathcal{N C}(T)$, by Proposition 17 .

This completes the proof of the soundness and completeness of the constraint characterization, i.e., Theorem 18.

\section{Polynomial inclusion checking via constraint satisfaction}

As shown in the previous section, type semantics can be characterized in terms of constraint satisfaction. As a consequence, type inclusion can also be characterized as constraint satisfaction, as follows.

\section{Corollary 25}

$$
\llbracket T \rrbracket \subseteq \llbracket U \rrbracket \Leftrightarrow T \models \mathcal{C C}(U) \wedge T \models \mathcal{O C}(U) \wedge T \models \mathcal{F C}(U)
$$

In the next sections we will show how each of the three properties in the right hand side can be verified in polynomial time.

\subsection{Polynomial checking of $T \models \mathcal{C C}(U)$}

We present here an algorithm to check whether $T=\mathcal{C C}(U)$ in $O\left(|T| *|U|+|U|^{2}\right)$ time.

In [8], we observed that co-occurrence constraints are mutually related in the same way as functional dependencies, if one switches the left sides with the right sides. More precisely, one can define a notion of "backward closure" as follows. 
Definition 26 For any $B \subseteq \Sigma$ and type $T$, we indicate with $\mathrm{BC}(B)_{T}$ the backward closure of $B$ with respect to $T$, and define it as:

$$
\mathrm{BC}(B)_{T}=\left\{a \in S(T) \mid T \models a^{+} \Leftrightarrow B^{+}\right\}
$$

Knowledge of $\mathrm{BC}(B)_{T}$ is sufficient to decide whether $T \models A^{+} \boxminus B^{+}$.

Lemma 27 For any $A, B, T$ :

$$
\begin{aligned}
& T \models A^{+} \mapsto B^{+} \\
& \Leftrightarrow \forall a \in(A \cap S(T)) . T \models a^{+} \mapsto B^{+} \\
& \Leftrightarrow(A \cap S(T)) \subseteq \mathrm{BC}(B)_{T}
\end{aligned}
$$

Proof. (1) $\Leftrightarrow(2): T \models A^{+} \Leftrightarrow B^{+}$

$\Leftrightarrow w \in \llbracket T \rrbracket \wedge w \models A^{+} \Rightarrow w \models B^{+}$

$\Leftrightarrow \forall a \in A . w \in \llbracket T \rrbracket \wedge w \models a^{+} \Rightarrow w \models B^{+}$

$\Leftrightarrow \forall a \in(A \cap S(T)) . w \in \llbracket T \rrbracket \wedge w \models a^{+} \Rightarrow w \models B^{+}$

$\Leftrightarrow \forall a \in(A \cap S(T)) . T \models a^{+} \Leftrightarrow B^{+}$.

$(2) \Leftrightarrow(3)$ : by definition of $\mathrm{BC}(B)_{T}$.

For $\mathrm{BC}(B)_{T}$, the following properties hold, which are proved by Lemma 28, but are anticipated here to to help the reader's intuition:

- transitivity: for any type $T=C\left[T_{1} \otimes T_{2}\right], \mathcal{C C}\left(T_{1} \otimes T_{2}\right)=I f_{T_{2}}\left(S^{+}\left(T_{1}\right) \Leftrightarrow\right.$ $\left.S^{+}\left(T_{2}\right)\right) \wedge \ldots$, hence:

$$
\neg \mathrm{N}\left(T_{2}\right) \wedge S\left(T_{2}\right) \subseteq \mathrm{BC}(B)_{T} \Rightarrow S\left(T_{1}\right) \subseteq \mathrm{BC}(B)_{T}
$$

- completeness: the repeated application of transitivity is a complete algorithm for computing $\mathrm{BC}(B)_{T}$.

According to these properties, $\mathrm{BC}(B)_{T}$ can be computed by the following algorithm, which "marks" a subterm $T^{\prime}$ of $T$ as soon as it can conclude that $S\left(T^{\prime}\right) \subseteq \mathrm{BC}(B)_{T}$ :

(1) mark all the leaves of the type whose symbol is in $B$, and visit the parse tree analyzing the parent of each marked node;

(2) if the parent of a marked $T_{2}$ with $\neg \mathrm{N}\left(T_{2}\right)$ is $T_{1} \otimes T_{2}$ (or $T_{2} \otimes T_{1}$ ), then mark it as well (thanks to transitivity) and continue the visit from its parent; 
(3) when both children of a subterm $T^{\prime}$ are marked, then $T^{\prime}$ is marked too and the visit continues from $T^{\prime}$.

Step (2) above does not mark $T_{1}$ and its descendants, but just $T_{1} \otimes T_{2}$. As a consequence, not every $a[m . . n]$ whose $a$ is in $\mathrm{BC}(B)_{T}$ is marked. Instead, $\mathrm{BC}(B)_{T}$ corresponds to the set of all $a[m . n]$ with at least one ancestor that is marked (Lemma 28).

Having presented the idea, we can now start the formal treatment. We first define the set $B_{T}^{\uparrow}$, that represents the subterms of $T$ that are marked by the above algorithm invoked with a $B$ set of symbols. In this definition, $T=C\left[T^{\prime}\right]$ means that $T^{\prime}$ is a subterm of $T$.

$$
\begin{array}{rlrl}
(\epsilon) & \text { for any } B, T & \epsilon \in B_{T}^{\uparrow} \\
(a[m . . n]) & T=C[a[m . . n]], a \in B & \Rightarrow a[m . . n] \in B_{T}^{\uparrow} \\
(+) & T=C\left[T_{1}+T_{2}\right], T_{1} \in B_{T}^{\uparrow}, T_{2} \in B_{T}^{\uparrow} \Rightarrow T_{1}+T_{2} \in B_{T}^{\uparrow} \\
(\otimes) & T=C\left[T_{1} \otimes T_{2}\right], T_{1} \in B_{T}^{\uparrow}, T_{2} \in B_{T}^{\uparrow} & \Rightarrow T_{1} \otimes T_{2} \in B_{T}^{\uparrow} \\
(\otimes \Leftrightarrow) & T=C\left[T_{1} \otimes T_{2}\right], \neg \mathrm{N}\left(T_{2}\right), T_{2} \in B_{T}^{\uparrow} & \Rightarrow T_{1} \otimes T_{2} \in B_{T}^{\uparrow} \\
(\otimes \Leftrightarrow) & T=C\left[T_{1} \otimes T_{2}\right], \neg \mathrm{N}\left(T_{1}\right), T_{1} \in B_{T}^{\uparrow} & \Rightarrow T_{1} \otimes T_{2} \in B_{T}^{\uparrow} \\
(!) & T=C\left[T_{1} !\right], T_{1} \in B_{T}^{\uparrow} & \Rightarrow T_{1} ! \in B_{T}^{\uparrow}
\end{array}
$$

Observe that $B_{T}^{\uparrow}$ is closed by type contexts, that is, for any type context $C[T]$, $T^{\prime} \in B_{T}^{\uparrow}$ implies $T^{\prime} \in B_{C[T]}^{\uparrow}$.

We use $S\left(B_{T}^{\uparrow}\right)$ to denote $\cup_{T^{\prime} \in B_{T}^{\uparrow}} S\left(T^{\prime}\right)$; the following lemma proves that $S\left(B_{T}^{\uparrow}\right)=$ $\mathrm{BC}(B)_{T}$.

Lemma 28 For each type T:

$$
\left(a \in S(T) \wedge T \models a^{+} \Leftrightarrow B^{+}\right) \Leftrightarrow a \in S\left(B_{T}^{\uparrow}\right)
$$

\section{Proof.}

We first consider the $\Rightarrow$ direction.

We prove the following property by induction on the structure of $T$ and by case analysis (the interesting case being $T=T_{1} \otimes T_{2}$ ):

$$
\begin{array}{ll}
\text { (1) } a \in S(T) \wedge T \models a^{+} \Leftrightarrow B^{+} & \Rightarrow a \in S\left(B_{T}^{\uparrow}\right) \\
\text { (2) }(\llbracket T \rrbracket \backslash\{\epsilon\}) \models B & \Rightarrow T \in B_{T}^{\uparrow}
\end{array}
$$


In line (2) we use the notation $W \models B$ to denote $\forall w \in W$. $w \models B$.

$\mathbf{T}=\epsilon$ :

1: Trivial, as $a \notin S(\epsilon)$.

2: Trivial, since $\epsilon \in B_{U}^{\uparrow}$ for each $U$.

$\mathbf{T}=\mathbf{b}[\mathbf{m} . . \mathbf{n}]:$

1: From $a \in S(T)$ we obtain that $a=b$. From $T \models b^{+} \Leftrightarrow B^{+}$we have that $b \in B$, hence $b[m . . n] \in B_{b[m . . n]}^{\uparrow}$.

2: From $(\llbracket T \rrbracket \backslash\{\epsilon\}) \models B$ we deduce $b \in B$, hence $b[m . . n] \in B_{b[m . . n]}^{\uparrow}$.

$\mathrm{T}=\mathrm{T}_{1}+\mathrm{T}_{2}$

Without loss of generality, we can assume that $a \in S\left(T_{1}\right)$.

1: From $T \models a^{+} \Leftrightarrow B^{+}$we obtain that $T_{1} \models a^{+} \Leftrightarrow B^{+}$, which, by induction, implies $a \in S\left(B_{T_{1}}^{\uparrow}\right)$, hence, by context closure, $a \in S\left(B_{T}^{\uparrow}\right)$.

2: From $(\llbracket T \rrbracket \backslash\{\epsilon\}) \models B$ we obtain that $\left(\llbracket T_{1} \rrbracket \backslash\{\epsilon\}\right) \models B$ and $\left(\llbracket T_{2} \rrbracket \backslash\{\epsilon\}\right) \models B$, which, by induction, imply $T_{1} \in B_{T_{1}}^{\uparrow}$ and $T_{2} \in B_{T_{2}}^{\uparrow}$, hence $\left(T_{1}+T_{2}\right) \in B_{T_{1}+T_{2}}^{\uparrow}$.

$\mathbf{T}_{1} \otimes \mathrm{T}_{2}$

Without loss of generality, we can assume that $a \in S\left(T_{1}\right)$.

1: We distinguish two cases:

$1.1 T_{2} \not \models B$, i.e. $\exists w_{2} \in \llbracket T_{2} \rrbracket$ such that $w_{2} \not \forall B$;

$1.2 T_{2}=B$, i.e. $\forall w_{2} \in \llbracket T_{2} \rrbracket w_{2} \models B$.

Case 1.1: We know that $\exists w_{2} \in \llbracket T_{2} \rrbracket$ such that $w_{2} \not \models B$, i.e. $w_{2}$ does not contain any symbol in $B$. For each string $w \in \llbracket T_{1} \rrbracket, w \cdot w_{2} \in \llbracket T_{1} \otimes T_{2} \rrbracket$ hence, by hypothesis, $w \cdot w_{2} \models a^{+} \Leftrightarrow B^{+}$. From $w_{2} \not \models B$, we deduce that, for any such $w, w \models a^{+} \Leftrightarrow B^{+}$, hence $T_{1} \models a^{+} \Leftrightarrow B^{+}$, hence $a \in S\left(B_{T_{1}}^{\uparrow}\right)$ (by induction), hence $a \in S\left(B_{T}^{\uparrow}\right)$ (by context closure).

Case 1.2: We know that $T_{2} \models B$, hence $\llbracket T_{2} \rrbracket \backslash\{\epsilon\} \models B$, hence, by induction, $T_{2} \in B_{T_{2}}^{\uparrow}$, which entails $T_{2} \in B_{T_{1} \otimes T_{2}}^{\uparrow}$ by context closure. The hypothesis $T_{2}=$ $B$ also implies that $\mathrm{N}\left(T_{2}\right)$ is false, hence, by definition of $B_{T_{1} \otimes T_{2}}^{\uparrow}$, from $T_{2} \in$ $B_{T_{1} \otimes T_{2}}^{\uparrow}$ we deduce $\left(T_{1} \otimes T_{2}\right) \in B_{T_{1} \otimes T_{2}}^{\uparrow}$, hence $S\left(T_{1} \otimes T_{2}\right) \subseteq S\left(B_{T_{1} \otimes T_{2}}^{\uparrow}\right)$, hence $a \in S\left(B_{T_{1} \otimes T_{2}}^{\uparrow}\right)$, since $a \in S\left(T_{1} \otimes T_{2}\right)$ by hypothesis. 
2: We have three cases:

$2.1 \mathrm{~N}\left(T_{1}\right)$ and $\mathrm{N}\left(T_{2}\right)$ both hold;

2.2 exactly one of $\neg \mathrm{N}\left(T_{1}\right)$ and $\neg \mathrm{N}\left(T_{2}\right)$ holds;

$2.3 \neg \mathrm{N}\left(T_{1}\right)$ and $\neg \mathrm{N}\left(T_{2}\right)$ both hold.

Case 2.1: $\epsilon \in \llbracket T_{1} \rrbracket$ implies that $\llbracket T_{2} \rrbracket \subseteq \llbracket T_{1} \otimes T_{2} \rrbracket$, and $\epsilon \in \llbracket T_{2} \rrbracket$ implies that $\llbracket T_{1} \rrbracket \subseteq \llbracket T_{1} \otimes T_{2} \rrbracket$, hence we can reason as in the case for $T_{1}+T_{2}$.

Case 2.2: assume that $\neg \mathrm{N}\left(T_{1}\right)$ and $\mathrm{N}\left(T_{2}\right)$. As in the previous case, $\epsilon \in \llbracket T_{2} \rrbracket$ implies that $\llbracket T_{1} \rrbracket \subseteq \llbracket T_{1} \otimes T_{2} \rrbracket$, hence $T_{1} \in B_{T_{1}}^{\uparrow}$ by induction, hence, by $\neg \mathrm{N}\left(T_{1}\right)$, $\left(T_{1} \otimes T_{2}\right) \in B_{T_{1} \otimes T_{2}}^{\uparrow}$.

Case 2.3: We prove that either $\left(\llbracket T_{1} \rrbracket \backslash\{\epsilon\}\right) \models B$ or $\left(\llbracket T_{2} \rrbracket \backslash\{\epsilon\}\right) \models B$, and the thesis follows as in case 2.2. Assume, for a contradiction, that both $\left(\llbracket T_{1} \rrbracket \backslash\right.$ $\{\epsilon\}) \forall \forall B$ and $\left(\llbracket T_{2} \rrbracket \backslash\{\epsilon\}\right) \not \forall B$ hold, then we have two non-empty words $w_{1}$ and $w_{2}$ such that $w_{1} \forall \forall B$ and $w_{2} \not \forall B$, and hence $w_{1} \cdot w_{2} \forall \neq B$, which contradicts the hypothesis, since $w_{1} \cdot w_{2} \in \llbracket T_{1} \otimes T_{2} \rrbracket \backslash\{\epsilon\}$.

$\mathbf{T}=\mathbf{T}_{1} !$

1: From $T \models a^{+} \Leftrightarrow B^{+}$we obtain that $T_{1} \models a^{+} \Leftrightarrow B^{+}$, which, by induction, implies $a \in S\left(B_{T_{1}}^{\uparrow}\right)$, hence $a \in S\left(B_{T}^{\uparrow}\right)$.

2: From $\llbracket T ! \rrbracket \backslash\{\epsilon\} \models B$ we obtain that $\llbracket T \rrbracket \backslash\{\epsilon\} \models B$, which, by induction, implies $T_{1} \in B_{T_{1}}^{\uparrow}$ hence $\left(T_{1} !\right) \in B_{T_{1}}^{\uparrow}$.

The $\Leftarrow$ direction is easy, and is proved by induction on the structure of $T$ and by case analysis.

In Figure 1 we present the algorithm BackwardClose(Type T, Set A) that marks any term in $A_{T}^{\uparrow}$. The algorithm works on a type $T$ that has been $\epsilon$ normalized, meaning that every subterm $\epsilon \otimes T$ or $T \otimes \epsilon$ is rewritten as $T$, and every $\epsilon+\epsilon$ is rewritten as $\epsilon$, until no such terms remain; this can be done in linear time, and allows $\epsilon$ terms not to be marked. A type $T$ is encoded by the arrays NodeOfSymbol[], Parent[], Oper[], LeftChild[], RightChild[]. For each symbol $a \in S(T)$, NodeOfSymbol $[a]$ is the only node $n d$ associated with a type $a[m . n]$, for some $m$ and $n$, and is null if no such node exists ${ }^{4}$; for each node $n$ in $T$, Parent $[n]$ is either null or a pair $\left(n_{p}, p o s\right) ; n_{p}$ is the parent of $n$, while pos is left if $n$ is the left child of $n_{p}$, and is right if it is the right child. The Oper[] array associates true ${ }_{2}$ to any + node where no child is $\epsilon$, true ${ }_{1}$

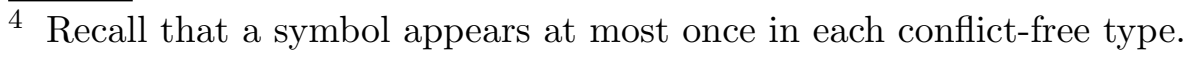


to a + node where one child is $\epsilon$. We use true to indicate that no constraint is associated with the node, while the pedices 2 and 1 refer to the number of children that have yet to be inserted into $A_{T}^{\uparrow}$ before the node can be inserted as well. An $\otimes$ node is associated with $\Leftrightarrow$ when no child is nullable, with $\Leftrightarrow$ when the right child only is nullable, $\Leftrightarrow$ when the left child only is nullable, with true $_{2}$ when both children are nullable. LeftChild[] and RightChild[] are defined in the obvious way.

The algorithm differs from ${ }_{-T}^{\uparrow}$ in that it explicitly marks every subterm of each term in $B_{T}^{\uparrow}$; this is performed by the MARKDown routine, which propagates the mark down until it meets a node that is marked already. In this way, all the children of any node in ToDo are marked, hence, thanks to the test in line 9, no node enters ToDo twice.

BACKWARDCLOSE first instantiates the above mentioned arrays in time $O(|T|)$. Then, the algorithm populates the ToDo list in time $O(|A|)$; ToDo contains all the nodes that must be visited. The body of the main loop (line 7 to line 23) needs constant time, apart from the calls to MARKDown which, collectively, mark less then $O(|T|)$ nodes. No node enters the ToDo list twice, hence the main loop takes time $O(|T|)$, hence the complexity of the algorithm is $O(|A|+|T|)$.

The algorithm immediately satisfies the invariant that, whenever a node enters ToDo, then the corresponding $T^{\prime}$ is in $A_{T}^{\uparrow}$, and that, whenever a node is marked, then one of its ancestors is in $A_{T}^{\uparrow}$. To prove that every node in $A_{T}^{\uparrow}$ is eventually inserted in ToDo, we proceed by induction on the size of the corresponding subtree $T^{\prime}$, assuming that all the subtrees of $T^{\prime}$ that are in $A_{T}^{\uparrow}$ are eventually inserted in ToDo.

We can now define the algorithm CoImplies (Figure 2) to check whether $T=$ $\mathcal{C C}(U)$. The algorithm invokes BACKWARDCLOSE once for each constraints $A^{+} \Leftrightarrow B^{+}$in $\mathcal{C C}(U)$, and verifies whether each $a$ in $A \cap S(T)$ belongs to $\mathrm{BC}(B)_{T}$. Each invocation of BACKWARdClose(Type T, Set B) is in $O(|T|+$ $|U|)$, and the test for $(A \cap S(T)) \subseteq \mathrm{BC}(B)_{T}$ is in $O(|U|)$, hence in $O(|T|+|U|)$. Since $\mathcal{C C}(U)$ contains at most $2 *|U|$ constraints, the complexity of CoImPLIES is $O\left(|T| *|U|+|U|^{2}\right)$.

\subsection{Polynomial checking of $T \models \mathcal{O C}(U)$}

In the previous section we have shown how to deduce all the co-occurrence constraints that are satisfied by a type $T$, by computing a sort of backward transitive closure on $\mathcal{C C}(T)$. Order constraints are much simpler, since $T$ does not satisfy any other order constraint apart from those that are actually present in $\mathcal{O C}(T)$; this is proved by the following theorem. 


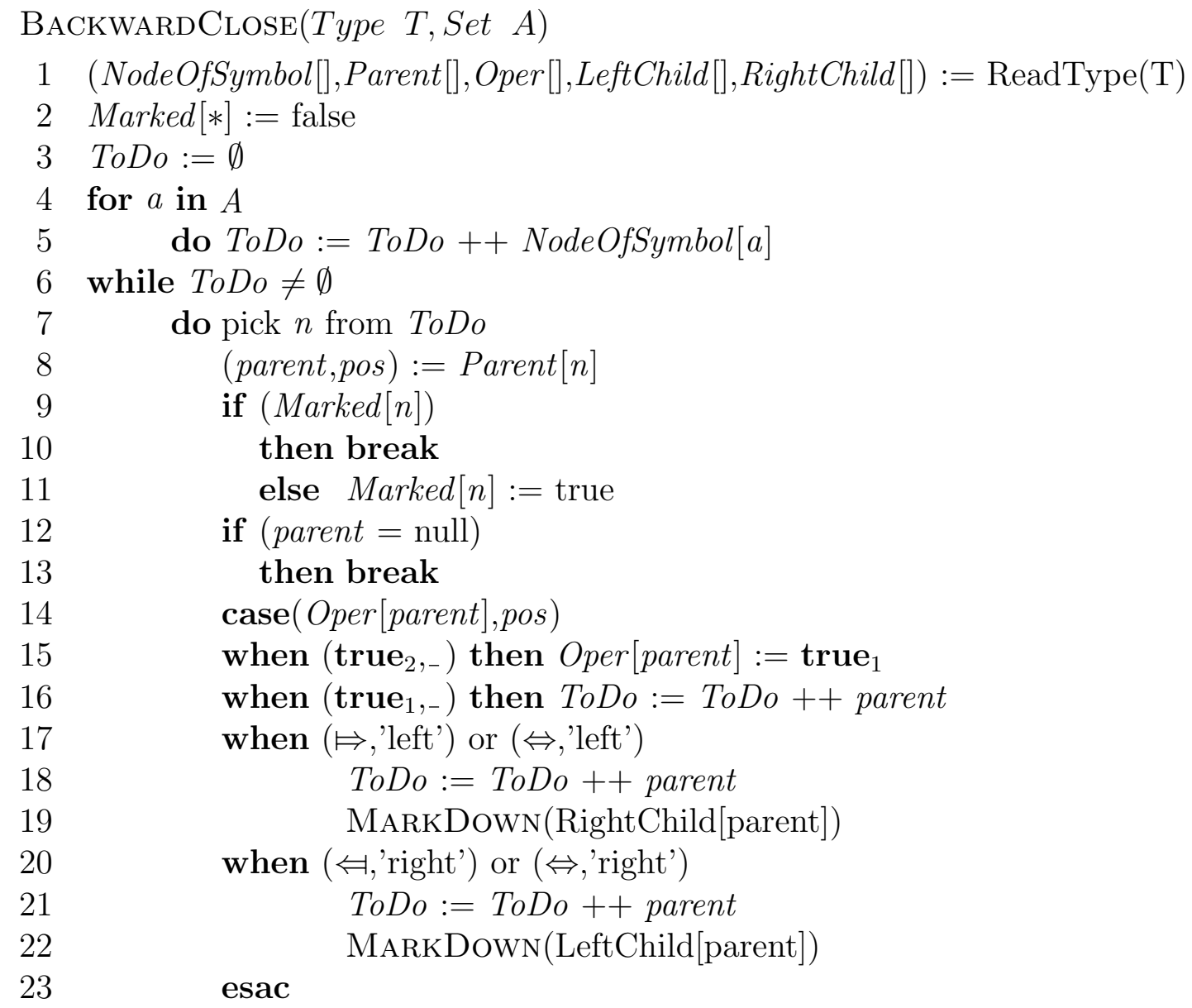

Fig. 1. Backward closure algorithm.

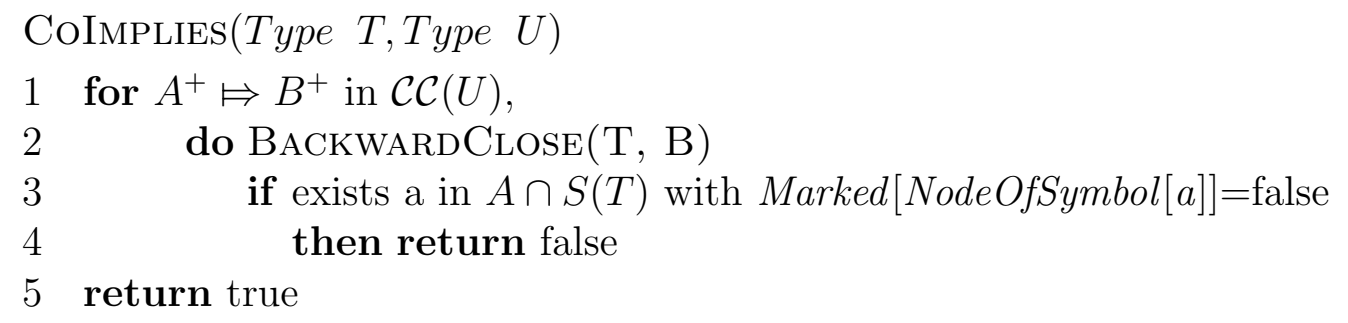

Fig. 2. Algorithm for implication of cooccurrence constraints.

Theorem 29 If $a \neq b$, then

$$
T \models a \prec b \Leftrightarrow(a \prec b \in \mathcal{O C}(T) \vee\{a, b\} \nsubseteq S(T))
$$

\section{Proof.}

The $\Leftarrow$ direction easily follows from the following facts:

- If $\{a, b\} \nsubseteq S(T)$, then each word $w$ in $T$ does not contain both $a$ and $b$; this means that $w \models a \prec b$; 
- If $a \prec b \in \mathcal{O C}(T)$, then $T \models a \prec b$ follows from the fact that $T \models \mathcal{O C}(T)$ (Theorem 18).

We focus now on the $\Rightarrow$ direction. We proceed by induction on the structure of $T$.

$\mathbf{T}=\epsilon:$

Trivial, as $S(T)=\emptyset$ entails $\{a, b\} \nsubseteq S(T)$.

$\mathbf{T}=\mathbf{c}[\mathbf{m} . . \mathbf{n}]:$

Trivial, we immediately have $\{a, b\} \nsubseteq S(T)$ since $a \neq b$.

$\mathbf{T}=\mathbf{T}_{1}+\mathbf{T}_{2}$

We suppose $\{a, b\} \subseteq S(T)$; the other case is trivial.

We have the following subcases:

Case 1. $\{a, b\} \subseteq S\left(T_{1}\right)$ or $\{a, b\} \subseteq S\left(T_{2}\right)$.

Case 2. $a \in S\left(T_{1}\right)$ and $b \in S\left(T_{2}\right)$, or vice versa.

For case 1 we only consider $\{a, b\} \subseteq S\left(T_{1}\right)$, the other one is similar. In this case we have $T_{1} \models a \prec b$. By induction we have $\left(a \prec b \in \mathcal{O C}\left(T_{1}\right) \vee\{a, b\} \nsubseteq\right.$ $S\left(T_{1}\right)$ ), and, therefore $a \prec b \in \mathcal{O C}\left(T_{1}\right)$ (as $\left.\{a, b\} \subseteq S\left(T_{1}\right)\right)$, which entails that $a \prec b \in \mathcal{O C}(T)$, since $\mathcal{O C}(T)=F \wedge \mathcal{O C}\left(T_{1}\right)$.

For case 2, we only consider $a \in S\left(T_{1}\right)$ and $b \in S\left(T_{2}\right)$, the other one being identical. In this case we have that $a \prec \succ b=(a \prec b \wedge b \prec a) \in \mathcal{O C}(T)$.

$\mathrm{T}_{1} \& \mathrm{~T}_{2}:$

We can have the following subcases:

Case 1. $\{a, b\} \subseteq S\left(T_{1}\right)$ or $\{a, b\} \subseteq S\left(T_{2}\right)$.

Case 2. $a \in S\left(T_{1}\right)$ and $b \in S\left(T_{2}\right)$, or vice versa.

Case 1 is similar to the corresponding one for case $T=T_{1}+T_{2}$, while we can exclude case 2 because of the hypothesis $T \models a \prec b$ and of Lemma 4(2).

$\mathrm{T}_{1} \cdot \mathrm{T}_{2}$ :

We can have the following subcases:

Case 1. $\{a, b\} \subseteq S\left(T_{1}\right)$ or $\{a, b\} \subseteq S\left(T_{2}\right)$. 
Case 2. $a \in S\left(T_{1}\right)$ and $b \in S\left(T_{2}\right)$, or vice versa.

Case 1 is similar to the corresponding one for case $T=T_{1}+T_{2}$.

For case 2 we can only have $a \in S\left(T_{1}\right)$ and $b \in S\left(T_{2}\right)$ (since the contrary would contradict $T \models a \prec b)$. For this case, we have $a \prec b \in \mathcal{O C}(T)$ by definition of $\mathcal{O C}(T)$.

T!:

This case easily follows by induction once we recall that $\llbracket T ! \rrbracket=\llbracket T \rrbracket \backslash\{\epsilon\}$, $S(T !)=S(T)$, and $\mathcal{O C}(T !)=\mathcal{O C}(T)$.

The previous theorem tells us that we can check $T=\mathcal{O C}(U)$ in $O(|\mathcal{O C}(T)|$. $|\mathcal{O C}(U)|)$ time, by checking whether each $a \prec b$ in $\mathcal{O C}(U)$ is also in $\mathcal{O C}(T)$, which gives us an upper bound $O\left(|T|^{2} \cdot|U|^{2}\right)$, but we can actually do much better. Indeed, we can perform the checking in $O\left(|T|+|U|^{2}\right)$ time. Before defining the algorithm, we make some preliminary observations.

For each pair of leaves $a[m . . n]$ and $b\left[m^{\prime} . . n^{\prime}\right]$ in the parse tree of $T$, let $L C A_{T}[a, b]$ be their common ancestor that is farthest from the root (the Lowest Common Ancestor). Order constraints correspond to the concatenation and union type operators:

- for each $a$ and $b$ in $S(T), a \prec \succ b \in \mathcal{O C}(T)$ iff $L C A_{T}[a, b]$ is labeled by + ;

- for each $a$ and $b$ in $S(T), a \prec b \in \mathcal{O C}(T)$ iff $L C A_{T}[a, b]=+$ or $a$ precedes $b$ in $T$ and $L C A_{T}[a, b]=\cdot$.

As a consequence, by Theorem 29, $T \models \mathcal{O C}(U)$ iff for each $a$ and $b$ in $S(U)$, such that $a$ precedes $b$ in $U$ :

- if $L C A_{U}[a, b]=+$ then either $a \notin S(T)$ or $b \notin S(T)$ or $L C A_{T}[a, b]=+$;

- if $L C A_{U}[a, b]=$. then either $a \notin S(T)$ or $b \notin S(T)$ or $L C A_{T}[a, b]=+$ or $\left(L C A_{T}[a, b]=\cdot\right.$ and $a$ precedes $b$ in $\left.T\right)$.

Hence, we can verify whether $T \models \mathcal{O C}(U)$ via the following algorithm. We first build an array $L C A_{T}[a, b]$, which associates each $a$ and $b$ in $S(T)$ with the operator that labels the LCA of $a$ and $b$ in $T$, and similarly for $U$; this can be done in linear time $O(|T|+|U|)$ [3]. We then scan all the ordered pairs $a, b$ of $S(U)$, checking the condition above, which can be done with $O\left(|U|^{2}\right)$ constant-time accesses to $L C A_{T}\left[_{-},,_{-}\right]$and $L C A_{U}\left[_{-},{ }_{-}\right]$, which gives a $O\left(|T|+|U|^{2}\right)$ algorithm. The resulting algorithm is defined in Figure 3. 


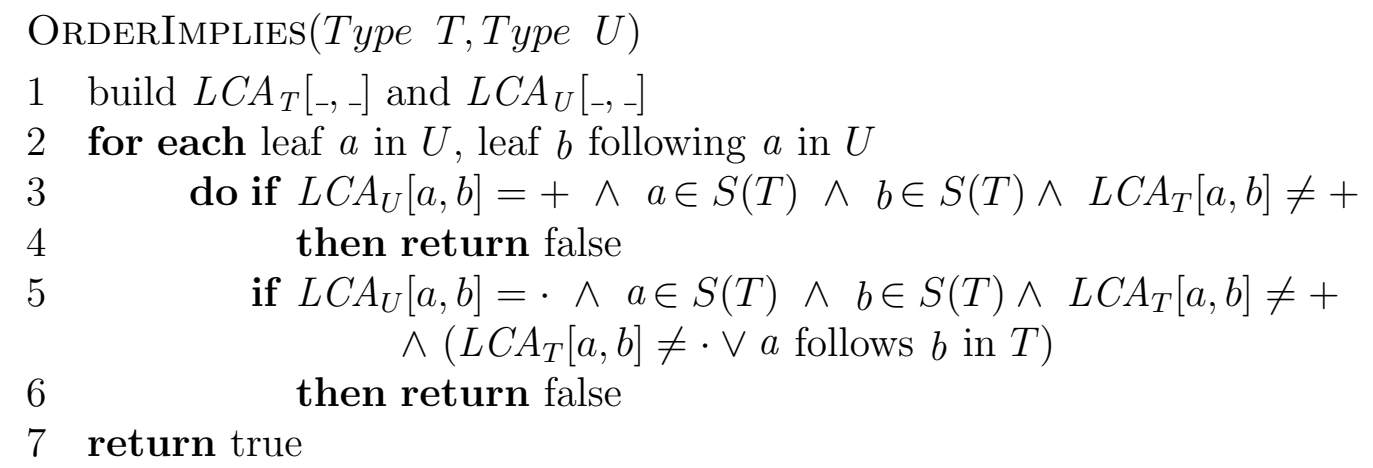

Fig. 3. Algorithm for implication of order constraints.

\subsection{Polynomial checking of $T \models \mathcal{F C}(U)$}

A systematic way to check flat constraint implication $T \models \mathcal{F C}(U)$ in polynomial time is directly illustrated by the following theorem, stating that, as expected, $T \models \mathcal{F C}(U)$ holds if and only if i) all cardinality constraints $a$ ? [m..n] of $T$ appear in $U$ as well, possibly in a relaxed form, and ii) if $\mathrm{N}\left(T_{1}\right)$ holds, then $\mathrm{N}\left(T_{2}\right)$ is true as well.

\section{Theorem 30}

$$
T \models \mathcal{F C}(U) \Leftrightarrow T \subseteq_{\text {flat }} U
$$

where $T \subseteq$ flat $U$ is defined as :

$$
\begin{aligned}
(a ?[m . . n] \in \operatorname{Atoms}(T) & \left.\Rightarrow \exists m^{\prime} \leq m, n^{\prime} \geq n . a\left[m^{\prime} . . n^{\prime}\right] \in \operatorname{Atoms}(U)\right) \\
\wedge(\mathrm{N}(T) & \Rightarrow \mathrm{N}(U))
\end{aligned}
$$

\section{Proof.}

Recall that $\mathcal{F C}(U)=S I f(U) \wedge \operatorname{upperS}(U) \wedge \operatorname{ZeroMinMax}(U)$.

Case $T \models \mathcal{F C}(U) \Leftarrow T \subseteq_{\text {flat }} U$

We first observe that $T \subseteq$ flat $U$ implies $S(T) \subseteq S(U)$. Recall that, by Theorem $24, T \models \operatorname{upperS}(T) \wedge \mathrm{ZMM-SIf}(T)$.

(1) $T \models \operatorname{SIf}(U)$ : if $\mathrm{N}(U)$, then $\operatorname{SIf}(U)=$ true, hence the statement is trivial. Assume that $\mathrm{N}(T)$ is false. We have that $T \models S I f(T)$ and $w \in \llbracket T \rrbracket$ imply $w \models S^{+}(T)$, hence $w \models S^{+}(U)$ by $S(T) \subseteq S(U)$.

(2) $T \models \operatorname{upperS}(U)$ : we must prove that $w \in \llbracket T \rrbracket$ and $w \models a^{+}$imply that $a \in S(U) ; T \models \operatorname{upperS}(T), w \in \llbracket T \rrbracket$ and $w \models a^{+}$imply that $a \in S(T)$, and $a \in S(U)$ follows from $S(T) \subseteq S(U)$.

(3) $T \models$ ZeroMinMax $(U)$ : we must prove that, for any $w \in \llbracket T \rrbracket$ and $a[m . . n] \in$ $\operatorname{Atoms}(U), w \models a$ ? [m..n]. If $w \models a^{+}$, then, by $T \models \operatorname{upperS}(T) \wedge \operatorname{ZeroMinMax}(T)$, 
$\exists m^{\prime}, n^{\prime}$ such that $a\left[m^{\prime} . . n^{\prime}\right] \in \operatorname{Atoms}(T)$ and $w=a ?\left[m^{\prime} . . n^{\prime}\right]$. By hypothesis, $\exists m^{\prime \prime} \leq m^{\prime}, n^{\prime \prime} \geq n^{\prime}$ such that $a\left[m^{\prime \prime} . . n^{\prime \prime}\right] \in \operatorname{Atoms}(U)$ hence, since $U$ is conflict-free, we have that $m^{\prime \prime}=m$ and $n^{\prime \prime}=n$. Hence, $w \models a$ ? $\left[m^{\prime} . . n^{\prime}\right]$ and $m \leq m^{\prime}, n^{\prime} \leq n$ imply $w \models a ?[m . . n]$.

Case $T \models \mathcal{F C}(U) \Rightarrow T \subseteq_{\text {flat }} U$.

We have that $T \models S I f(U) \wedge \operatorname{upperS}(U) \wedge \operatorname{ZeroMinMax}(U)$. In particular, $T \models \operatorname{upperS}(U)$ means that, for each $w \in \llbracket T \rrbracket$, we have $w \models \operatorname{upperS}(U)$; this entails $S(T) \subseteq S(U)$, so

$$
\forall a ?[m . . n] \in \operatorname{Atoms}(T) . \exists a ?\left[m^{\prime} . . n^{\prime}\right] \in \operatorname{Atoms}(U)
$$

hence it remains to prove that it is always the case that $m^{\prime} \leq m$ and $n^{\prime} \geq n$. This follows by observing that, if we assume $n^{\prime}<n$ or $m^{\prime}>m$, then the hypothesis $T \models \mathcal{F C}(U)$ is contradicted. This is due to the following fact: if $a ?[m . . n] \in \operatorname{Atoms}(T)$ and $n \neq *$, then we have two words $w_{m}$ and $w_{n}$ in $\llbracket T \rrbracket$ such that they contain $m$ and $n$ occurrences of $a$ respectively; if $n=*$ and $n^{\prime} \neq *$ (the remaining cases are trivial), then there exists $w_{n}$ in $\llbracket T \rrbracket$, such that it contains more than $n^{\prime}$ occurrences of $a$. For these two words $w_{n}$ and $w_{m}$ we have that, under the hypothesis $\left(n^{\prime}<n\right.$ or $\left.m^{\prime}>m\right)$, either $w_{m} \models \operatorname{ZeroMinMax}(U)$ or $w_{n} \models \operatorname{ZeroMinMax}(U)$ does not hold, and so $T \models \operatorname{ZeroMinMax}(U)$ would be contradicted.

It remains to prove that $\mathrm{N}(T) \Rightarrow \mathrm{N}(U)$. Assume that $\mathrm{N}(T)$ holds (the other case is trivial). This means that $\epsilon \in \llbracket T \rrbracket$ and $\epsilon=S I f(U)$, by hypothesis.

If $S(U)=\emptyset$ (that is $U$ is equivalent to $\epsilon$ ), then $\mathrm{N}(U)$ holds and the thesis is proved. If $S(U) \neq \emptyset$, then it must be $S I f(U)=$ true, because otherwise $\epsilon \models S I f(U)$ would not hold, thus contradicting the hypothesis $T \models \mathcal{F C}(U)$

\subsection{Wrapping up: the inclusion checking algorithm}

We have provided algorithms to check $T \models \mathcal{C C}(U), T \models \mathcal{O C}(U)$ and $T \models$ $\mathcal{F C}(U)$. They can be used to build the inclusion checking algorithm illustrated in Figure 4. It first verifies whether $T \models \mathcal{F C}(U)$, in time $O(|T|+|U|)$ in the size of $T$ and $U$, as illustrated in Section 4.3. Then the algorithm invokes CoImplies and OrderImplies; the $O\left(|T| *|U|+|U|^{2}\right)$ time complexity of the first dominates the $O\left(|T|+|U|^{2}\right)$ complexity of the second, hence this is the cost of the whole algorithm. 


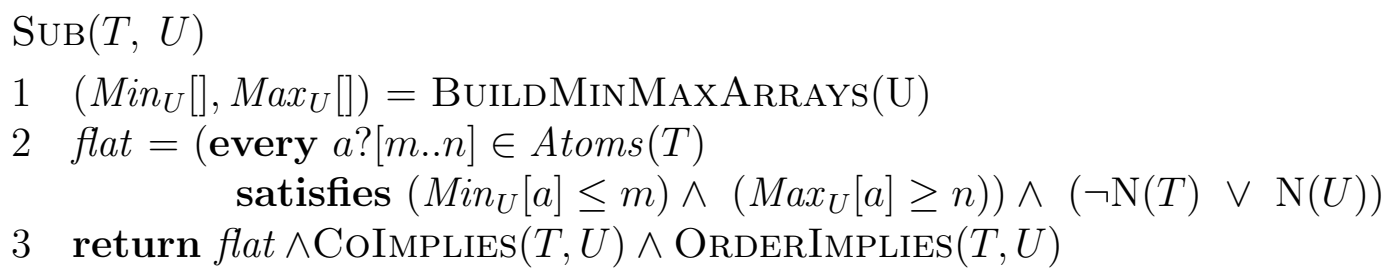

Fig. 4. Inclusion checking algorithm.

\section{Complexity of Binary Intersection}

Checking the non-emptiness of binary intersection of REs is typically cheaper than inclusion checking, since intersection ${ }^{5}$ is checked through automata product, while inclusion corresponds to automata complement plus product.

The situation is different for conflict-free types: while inclusion is in PTIME, intersection of two conflict-free expressions is NP-complete. This result is quite surprising, and it suggests that it makes sense to study such types with an approach that is not based on automata.

Interestingly, as shown by our proof, the \& operator alone makes binary intersection NP-hard, even without counting or Kleene star.

We first prove that non-emptiness is in NP, which is simple, since one has just to guess a word and check whether it is in both types; we have still to show that the word to guess is not big. To this aim, we show that any word $w \in(\llbracket T \rrbracket \cap \llbracket U \rrbracket)$ can be cut down to a word that is smaller than $|T|+|U|$ and is still in $\llbracket T \rrbracket \cap \llbracket U \rrbracket$. To achieve this bound, we represent each word abbbcc as $a^{1} b^{3} c^{2}$, as specified by the following definition.

Definition 31 (Binary word representation) A binary word representation is a sequence of symbol-integer pairs, written $a_{1}^{m_{1}} \ldots a_{k}^{m_{k}}$, where each $m_{i}>0$, and each pair $a_{i}^{m_{i}}$ represents a sequence of $m_{i}$ copies of $a_{i}$.

We now show how to reduce $w \in \llbracket T \rrbracket$ into a short form that is still in $\llbracket T \rrbracket$. We consider a set $f$ of pairs $(a, m)$, that defines a partial function from $\Sigma$ to $\mathbb{N}$. We define a function $\operatorname{Stem}(w, f)$ that deletes from $w$ the symbols that are not in $f$, and keeps just one repeated occurrence $a^{f(a)}$ for the others, so that $|\operatorname{Stem}(w, f)|<|f|$.

Definition $32(\operatorname{Stem}(w, f))$ For any word $w$ and set of pairs $f=\left\{\left(a_{1}, m_{1}\right), \ldots,\left(a_{k}, m_{k}\right)\right\}$, Stem $(w, f)$ is defined as follows, where, in the

\footnotetext{
$\overline{5}$ In this section we use intersection as an abbreviation for "non-emptiness of binary intersection"
} 
second line, $a^{f(a)}$ is defined as $\epsilon$ if a is not in $f$.

$$
\begin{aligned}
& \operatorname{Stem}(\epsilon, f) \quad={ }_{\text {def }} \epsilon \\
& \operatorname{Stem}\left(a^{n} \cdot w, f\right)=\operatorname{def}^{f(a)} \cdot \operatorname{Stem}(w, f \backslash(a, f(a)))
\end{aligned}
$$

Lemma 33 specifies that $w \in \llbracket T \rrbracket$ implies $\operatorname{Stem}(w, f) \in \llbracket T \rrbracket$, when $f$ leaves one copy of each character, and assigns acceptable exponents.

Lemma $33(\operatorname{Stem}(w, f) \in \llbracket T \rrbracket)$

$$
\begin{aligned}
& w \in \llbracket T \rrbracket \\
& \wedge S(f) \supseteq S(w) \\
& \wedge\left((a, f(a)) \in f \Rightarrow a^{f(a)} \models \operatorname{ZeroMinMax}(T)\right) \\
& \Rightarrow \operatorname{Stem}(w, f) \in \llbracket T \rrbracket .
\end{aligned}
$$

Proof. We prove that $\operatorname{Stem}(w, f)$ satisfies $\operatorname{SIf}(T), \operatorname{ZeroMinMax}(T), \operatorname{upperS}(T)$, $\mathcal{C C}(T), \mathcal{O C}(T)$, and we conclude by Theorem 18. By $S(f) \supseteq S(w)$, we deduce that no symbol is removed from $w$, hence $w \models S I f(T)$ implies that $\operatorname{Stem}(w, f) \models \operatorname{SIf}(T)$; for the same reason, $w \models \mathcal{C C}(T)$ implies that $\operatorname{Stem}(w, f) \models$ $\mathcal{C C}(T)$. From $a^{f(a)} \models \operatorname{ZeroMinMax}(T)$ we deduce that $\operatorname{Stem}(w, f) \models \operatorname{ZeroMinMax}(T)$. From $S(\operatorname{Stem}(w, f)) \subseteq S(w)$ we deduce that $\operatorname{Stem}(w, f) \models \operatorname{upperS}(T)$. Finally, if a pair of symbols $a$ and $b$ appear in $\operatorname{Stem}(w, f)$ in this order, then the first occurrence of $a$ precedes the first occurrence of $b$ in $w$; hence, $w \models \mathcal{O C}(T)$ implies that $\operatorname{Stem}(w, f) \models \mathcal{O C}(T)$.

Theorem 34 (Upper bound) Non emptiness of the intersection of two conflictfree types is in NP.

Proof. We prove that, if a word $w$ is in $\llbracket T \rrbracket \cap \llbracket U \rrbracket$, then a word $w^{\prime}$ whose binary representation is shorter than $|T|+|U|$ is in $\llbracket T \rrbracket \cap \llbracket U \rrbracket$ as well. The result follows, since non-emptiness can be checked by guessing a word shorter than $|T|+|U|$ and checking that this word satisfies all the constraints of $T$ and $U$, which is clearly in PTIME.

Define $f$ as follows:

$$
\begin{gathered}
f=\left\{\left(a, \max \left(m, m^{\prime}\right)\right) \mid a[m . . n] \in \operatorname{Atoms}(T), a\left[m^{\prime} . . n^{\prime}\right] \in \operatorname{Atoms}(U),\right. \\
\left.\max \left(m, m^{\prime}\right) \leq \min \left(n, n^{\prime}\right)\right\}
\end{gathered}
$$

We show that $f$ satisfies the conditions of Lemma 33 for both $T$ and $U$. Let $a \in S(w)$; from $w \models \operatorname{upperS}(T)$ and $w \models \operatorname{upperS}(U)$ we deduce that $a[m . . n] \in$ 
$\operatorname{Atoms}(T)$ and $a\left[m^{\prime} . . n^{\prime}\right] \in \operatorname{Atoms}(U)$. From $a \in S(w), w=\operatorname{ZeroMinMax}(T)$ and $w \models \operatorname{ZeroMinMax}(U)$, we deduce that $\max \left(m, m^{\prime}\right) \leq k \leq \min \left(n, n^{\prime}\right)$, where $k=|w|_{S(\{a\})} \mid$, hence $S(f) \supseteq S(w)$. The conditions $(a, f(a)) \in f \Rightarrow$ $a^{f(a)} \models \operatorname{ZeroMinMax}(T)$ and $(a, f(a)) \in f \Rightarrow a^{f(a)} \models \operatorname{ZeroMinMax}(U)$ hold by construction. By Lemma 33, we conclude that $\operatorname{Stem}(w, f) \in \llbracket T \rrbracket \cap \llbracket U \rrbracket$. By construction, $|\operatorname{Stem}(w, f)| \leq|f| \leq(|T|+|U|)$.

We can now prove that the problem is NP-hard, by reduction to 3-SAT.

Theorem 35 (Lower bound) Emptiness of the intersection of two conflictfree types is NP-hard, even if the types do not use counting and concatenation.

Proof. Consider $m$ boolean variables $x_{1}, \ldots, x_{m}$ and a formula $\phi=\left(a_{1,1} \vee\right.$ $\left.a_{2,1} \vee a_{3,1}\right) \wedge \ldots \wedge\left(a_{n, 1} \vee a_{n, 2} \vee a_{n, 3}\right)$ where each literal $a_{i, j}$ is either a variable $x_{l}$ or a negated variable $\neg x_{l}$; 3SAT is the problem of deciding, for such a $\phi$, whether an assignment of boolean values to $x_{1}, \ldots, x_{m}$ exists that satisfies the formula. Satisfiability of $\phi$ can be encoded as the intersection of two conflictfree types $T_{1}$ and $T_{2}$ as exemplified below. Both types have one symbol for each occurrence of a literal in $\phi$, hence their size is linear in $|\phi|$. Observe that each line in $T_{2}$ sums the positive occurrences of one variable with the negative ones, hence making the two mutually incompatible.

$$
\begin{aligned}
\phi= & \left(x_{1} \vee x_{2} \vee x_{3}\right) \wedge\left(\neg x_{1} \vee x_{3} \vee x_{4}\right) \wedge\left(\neg x_{2} \vee \neg x_{3} \vee \neg x_{4}\right) \wedge\left(\neg x_{1} \vee \neg x_{3} \vee x_{4}\right) \\
T_{1}= & \left(a_{1,1}+a_{1,2}+a_{1,3}\right) \&\left(a_{2,1}+a_{2,2}+a_{2,3}\right) \&\left(a_{3,1}+a_{3,2}+a_{3,3}\right) \&\left(a_{4,1}+a_{4,2}+a_{4,3}\right) \\
T_{2}= & \left(\left(a_{1,1} ?\right)+\left(a_{2,1} ? \& a_{4,1} ?\right)\right) \\
& \&\left(\left(a_{1,2} ?\right)+\left(a_{3,1} ?\right)\right) \\
& \&\left(\left(a_{1,3} ? \& a_{2,2} ?\right)+\left(a_{3,2} ? \& a_{4,2} ?\right)\right) \\
& \&\left(\left(a_{2,3} ? \& a_{4,3} ?\right)+\left(a_{3,3} ?\right)\right)
\end{aligned}
$$

A formal definition of the translation can be easily produced.

The formula $\phi$ is satisfiable iff it has a witness, i.e. a choice of literal instances, one from each factor, such that not two instances are contradictory, i.e. if $x_{i}$ is chosen in a factor then $\neg x_{i}$ is not chosen in any other factor.

Any element of $T_{1}$ corresponds to a choice of literal instances, one from each factor. If the same list also belongs to $T_{2}$, then the choice is not contradictory: if the word contained both a true and a false instance of the same variable, it should contain two symbols that come from the left and from the right side of a + operator in the line of $T_{2}$ that corresponds to that variable. Hence, words in $\llbracket T_{1} \rrbracket \cap \llbracket T_{2} \rrbracket$ correspond to witnesses for $\phi$. 


\section{Related Work}

This paper is an extended and revised version of [8]; in particular, while [8] provided a cubic inclusion algorithm, we provide here an algorithm that is quadratic, and whose crucial component (co-occurrence constraint implication) is simpler.

The properties of unordered XML types have been studied in several recent papers. In [6], the authors discuss the techniques and heuristics they used in implementing a type-checker, based on sheaves automata with Presburger arithmetic, for unordered XML types. The type language is an extension of the language we are considering here, and shares a similar restriction on the use of repetition types. The main purpose of the paper is to address scalability problems that naturally arise when working on XML types; as a consequence, they describe effective heuristics that improve scalability, but do not affect computational complexity.

Restrictions to regular expression languages that are similar to ours have been proposed many times. For example, conflict-free REs appear as "conflict-free DTDs" in the context of well-typed XML updates in [2], as "duplicate-free DTDs" in the context of path inclusion in [13], and as "single occurrence REs" in the context of DTD inference in [5]. The same restriction that we pose on Kleene-star can be found, for example, in [6]. Chain Regular Expressions (CHARE's) [5,7] are also strictly related. They are defined as concatenations of factors, where each factor has a shape $\left(a_{1}+\ldots+a_{n}\right),\left(a_{1}+\ldots+a_{n}\right)$ ?, $\left(a_{1}+\ldots+a_{n}\right)^{*}$ or $\left(a_{1}+\ldots+a_{n}\right)^{+}$. As we discussed in Section 2.1, we can express in our language each of these classes of factors. Simple expressions [4] have a more general syntax than CHAREs but the same expressive power, hence can still be managed through our approach.

We have cited paper [7] many times, where the complexity of type inclusion is studied for many different dialects of REs with interleaving and/or counting, showing that inclusion complexity is almost invariably EXPSPACE-complete. In particular, this is shown to hold for chain-REs with counting, which are concatenations of CHARE factors, as defined above, and counting factors $\left(a_{1}+\right.$ $\left.\ldots+a_{k}\right)[m . . n]$ (with $n \neq *$ and $m \geq 0$ ), with no interleaving operator. In a sense, this hints that the conflict-free restriction, rather than the Kleene-star restriction, is crucial for our PTIME result. In the same paper, the authors introduce a sublanguage of CHAREs with PTIME inclusion, but that fragment is quite trivial, since it only includes counting factors $\left(a_{1}+\ldots+a_{k}\right)[m . . n]$, with the further restriction that $m>0$ and $n \neq *$, hence cannot express neither optionality nor unbounded repetition (neither ${ }^{*}$ nor ${ }^{+}$). 


\section{Conclusions}

Inclusion for REs with interleaving, counting, or both, is EXPSPACE-complete, even if we consider the restricted subclass of CHAREs (with counting) [11,7]. This result easily extends to XML types featuring these operators. We have introduced here a restricted class of REs with interleaving and counting. Our restriction is severe, but it seems to match reasonably well the measured features of actual DTDs and XSDs found on the web, and is extremely easy to define and verify. For this class of REs, we have proved that inclusion can be decided in quadratic time, a complexity that is surprising low, and trivially extends to DTDs and XSDs that use REs of this class for their content models. In particular, we exhibited a quadratic algorithm for content-model inclusion. We also proved that intersection of two conflict-free types has not the same complexity as inclusion (unless $\mathrm{P}=\mathrm{NP}$ ) but is, quite surprisingly, NP-complete.

Our result is based on the transformation of our REs into sets of constraints which completely characterize the expressions and are easy to manipulate. We believe that this constraint-based approach could be fruitfully used for other analysis tasks, such as, for example, type normalization or path minimization under a DTD.

As a proof of concept of our approach, we implemented our algorithm and tested it against a set of randomly generated regular expression types. Our preliminary experimental evaluation showed that inclusion checking is very fast and is always performed in less than a second, even on very large types (e.g., more than 1000 terms). More information about our implementation can be found at http://www.di.unipi.it/ sartiani/projects/xelf.html.

\subsection{Acknowledgments}

We thank the anonymous referees of DBPL'07 and IS for their constructive comments and suggestions. We would also like to thank Luca Pardini for his work on the implementation of our approach.

\section{References}

[1] S. Amer-Yahia, S. Cho, L. V. S. Lakshmanan, D. Srivastava, Minimization of tree pattern queries., in: Proceedings of the 2001 ACM SIGMOD International Conference on Management of Data, Santa Barbara, California, May 21-24, 2001, 2001. 
[2] D. Barbosa, A. O. Mendelzon, L. Libkin, L. Mignet, M. Arenas, Efficient incremental validation of XML documents., in: Proceedings of the 20th International Conference on Data Engineering, ICDE 2004, 30 March - 2 April 2004, Boston, MA, USA, IEEE Computer Society, 2004.

[3] M. A. Bender, M. Farach-Colton, The LCA problem revisited., in: G. H. Gonnet, D. Panario, A. Viola (eds.), LATIN, vol. 1776 of Lecture Notes in Computer Science, Springer, 2000.

[4] G. J. Bex, F. Neven, J. V. den Bussche, DTDs versus XML Schema: A practical study, in: Proceedings of the Seventh International Workshop on the Web and Databases, WebDB 2004, June 17-18, 2004, Maison de la Chimie, Paris, France, Colocated with ACM SIGMOD/PODS 2004, 2004.

[5] G. J. Bex, F. Neven, T. Schwentick, K. Tuyls, Inference of concise DTDs from XML data, in: U. Dayal, K.-Y. Whang, D. B. Lomet, G. Alonso, G. M. Lohman, M. L. Kersten, S. K. Cha, Y.-K. Kim (eds.), Proceedings of the 32nd International Conference on Very Large Data Bases, Seoul, Korea, September 12-15, 2006, ACM, 2006.

[6] J. N. Foster, B. C. Pierce, A. Schmitt, A logic your typechecker can count on: Unordered tree types in practice, in: Workshop on Programming Language Technologies for XML (PLAN-X), informal proceedings, 2007.

[7] W. Gelade, W. Martens, F. Neven, Optimizing schema languages for XML: Numerical constraints and interleaving, in: T. Schwentick, D. Suciu (eds.), Proceedings of the 11th International Conference on Database Theory - ICDT 2007, Barcelona, Spain, January 10-12, 2007, vol. 4353 of Lecture Notes in Computer Science, Springer, 2007.

[8] G. Ghelli, D. Colazzo, C. Sartiani, Efficient inclusion for a class of XML types with interleaving and counting, in: M. Arenas, M. I. Schwartzbach (eds.), Proceedings of the 11th International Symposium on Database Programming Languages, DBPL 2007, Vienna, Austria, September 23-24, 2007, Revised Selected Papers, vol. 4797 of Lecture Notes in Computer Science, Springer, 2007.

[9] G. Ghelli, D. Colazzo, C. Sartiani, Linear time membership for a class of xml types with interleaving and counting, in: PLAN-X 2008, Programming Language Technologies for XML, An ACM SIGPLAN Workshop colocated with POPL 2008, San Francisco, California, USA, January 9, 2008, 2008.

[10] W. Martens, F. Neven, T. Schwentick, Complexity of decision problems for simple regular expressions., in: J. Fiala, V. Koubek, J. Kratochvíl (eds.), Proceedings of the 29th International Symposium on Mathematical Foundations of Computer Science, MFCS 2004, Prague, Czech Republic, August 22-27, 2004, vol. 3153 of Lecture Notes in Computer Science, Springer, 2004.

[11] A. J. Mayer, L. J. Stockmeyer, Word problems - this time with interleaving, Inf. Comput. 115 (2) (1994) 293-311. 
[12] H. S. Thompson, D. Beech, M. Maloney, N. Mendelsohn, XML Schema Part 1: Structures Second Edition, Tech. rep., World Wide Web Consortium, W3C Recommendation (Oct 2004).

[13] P. T. Wood, Containment for XPath fragments under dtd constraints, in: D. Calvanese, M. Lenzerini, R. Motwani (eds.), Proceedings of the 9th International Conference on Database Theory - ICDT 2003, Siena, Italy, January 8-10, 2003, vol. 2572 of Lecture Notes in Computer Science, Springer, 2003. 